\title{
Kernos
}

Revue internationale et pluridisciplinaire de religion

grecque antique

32 | 2019

Varia

\section{Variations dans le paysage sacré de Pergame : l'Asklépieion et le temple de la terrasse du théâtre}

\section{Stefano Caneva}

\section{(2) OpenEdition \\ Journals}

Édition électronique

URL : https://journals.openedition.org/kernos/3136

DOI : 10.4000/kernos.3136

ISSN : 2034-7871

Éditeur

Centre international d'étude de la religion grecque antique

Édition imprimée

Date de publication : 1 décembre 2019

Pagination : 151-181

ISBN : 978-2-87562-229-7

ISSN : 0776-3824

Référence électronique

Stefano Caneva, «Variations dans le paysage sacré de Pergame : I'Asklépieion et le temple de la terrasse du théâtre », Kernos [En ligne], 32 | 2019, mis en ligne le 01 octobre 2021, consulté le 01 février 2022. URL : http://journals.openedition.org/kernos/3136; DOI : https://doi.org/10.4000/kernos. 3136

Ce document a été généré automatiquement le 1 février 2022.

Kernos 


\title{
Variations dans le paysage sacré de Pergame : l'Asklépieion et le temple de la terrasse du théâtre
}

\author{
Stefano Caneva
}

La recherche à l'origine de cette contribution s'insère dans mon projet Practicalities of Hellenistic Ruler Cult (PHRC), qui a été subventionné, pendant les années 2015-2017, par une bourse Marie Curie Piscopia à l'Université de Padoue. La phase de finalisation de cet article a coïncidé avec la première année de mon mandat de chargé de recherche F.R.S.-FNRS à l'Université de Liège (2017-2020). Je tiens à remercier Vinciane Pirenne-Delforge ainsi que les lecteurs anonymes de Kernos pour leurs observations à une version préliminaire de cette contribution. Je reste évidemment le seul responsable de toute faute ou imprécision restée dans le texte.

\section{Le paysage sacré de Pergame dans la « longue période hellénistique »}

1 L'attention croissante attribuée pendant les dernières décennies à l'histoire de la période hellénistique a amené les savants à partager la nécessité, déjà aperçue par les épigraphistes spécialisés dans les institutions de la polis, d'une périodisation moins générique que celle imposée par les biographies d'Alexandre et Auguste, et ceci à la fois à l'intérieur et au-delà des limites classiques (323-31 av. J.-C.) de la période concernée.

D'un côté, une distinction s'est imposée entre une " haute » et une "basse " période hellénistique dont la jonction se situerait, avec des différences régionales, au cours du II $^{\mathrm{e}} \mathrm{s}$. av. J.-C. Cette distinction permet de rendre compte de deux facteurs strictement liés : la substitution de Rome aux royaumes nés des conquêtes d'Alexandre dans le rôle d'arbitre des relations internationales dans la Méditerranée orientale ; l'affirmation, au sein de la polis, d'une classe dirigeante composée d'un nombre limité de familles capables de remplacer l'évergétisme royal en déclin et d'agir comme médiateurs entre les instances locales et le pouvoir romain ${ }^{1}$. D'un autre côté, les tendances caractérisant 
la vie des cités à la basse période hellénistique ne s'achèvent pas lorsque l'organisation institutionnelle de Rome passe de l'ordre républicain au Principat. C'est pourquoi on constate l'utilisation de plus en plus fréquente d'une périodisation hellénistique longue, embrassant les trois siècles après Alexandre (ou au moins la période à partir du II $^{\mathrm{e}}$ siècle av. J.-C.) ainsi que la haute période impériale, avant l'avènement des Sévères ${ }^{2}$.

En partant de ce grand cadre général, cette contribution vise à explorer l'interaction entre les changements de l'histoire politique et sociale dans cette «longue période hellénistique » et l'évolution du paysage sacré de Pergame (Fig. 1) ${ }^{3}$, à travers deux cas d'étude : l'Asklépieion situé dans la vallée du Kaïkos, en face de Pergame, et le temple de la terrasse du théâtre, sur le versant ouest de la citadelle. Bien que ces deux sanctuaires aient eu une histoire distincte, le choix de les reconsidérer ensemble ressort de deux raisons: d'abord, parce que dans l'histoire des études, les tentatives d'identifier le dieu vénéré dans le temple de la terrasse du théâtre ont fait référence à plusieurs reprises au culte d'Asklépios; ensuite, parce qu'au-delà des différences évidentes, l'histoire de ces deux lieux de culte exemplifie l'organisation du paysage sacré de Pergame pendant la longue période hellénistique. Cette histoire se caractérise par une alternance entre deux phases " chaudes ", caractérisée par une accélération du processus de monumentalisation, et un intervalle "froid " pendant lequel le rapport entre la communauté et son paysage sacré prend davantage l'aspect d'un effort (qui ne se révèle pas toujours efficace) de préservation, sans que l'on puisse identifier des initiatives de renouvellement d'envergure ${ }^{4}$.

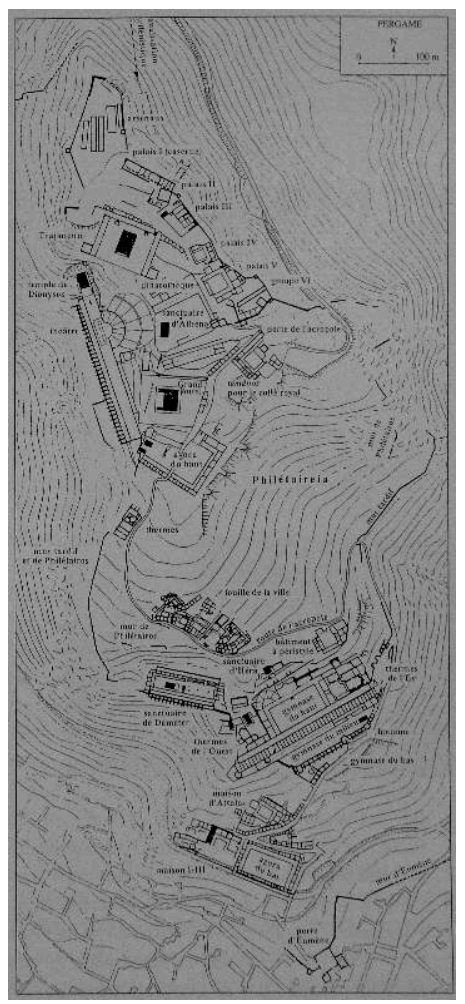

Fig. 1. Plan de la cité de Pergame avec ses sanctuaires, d'après auerrel (2005).

Les deux phases de monumentalisation se situent respectivement au moment où Pergame est la capitale de la dynastie attalide et à l'époque où la cité, désormais bien intégrée dans l'Empire, est en compétition pour son prestige avec d'autres cités de la province d'Asie, en particulier Smyrne et Éphèse. Il existe deux différences capitales 
entre ces deux phases. En premier lieu, à l'époque attalide, la monumentalisation du paysage sacré de Pergame se fait à l'initiative d'une dynastie locale visant à façonner sa propre capitale de manière conforme à ses aspirations géopolitiques, alors que, sous l'Empire, ce processus s'insère dans une dynamique d'interaction entre une cité provinciale et ses maîtres géographiquement éloignés. Cette situation active une logique de compétition entre Pergame et d'autres cités de la province d'Asie pour l'obtention d'un accès favorisé à l'attention de l'empereur. La multiplication des temples néocoriques du culte impérial s'intègre à plein titre dans cette logique. Deuxièmement - on peut considérer cette différence comme une conséquence de la première -, le développement monumental de Pergame sous les Attalides embrasse, bien qu'avec des nuances internes et avec des priorités dictées par les intérêts de la dynastie, la totalité des lieux de cultes (et des autres espaces publics) de la cité ; à la période impériale, en revanche, le procès de monumentalisation de l'espace public se focalise principalement sur les éléments qui peuvent assurer le prestige international de Pergame au sein de l'Empire : les temples du culte impérial, pour lesquels le permis accordé par l'empereur concrétise la faveur qu'il a envers la cité, le nouveau temple des divinités isiaques dans la vallée (milieu du $\mathrm{II}^{\mathrm{e}} \mathrm{s}$. ap. J.-C.) $)^{5}$ et l'Asklépieion, le seul sanctuaire pergaménien capable de capturer à une échelle internationale l'attention du tourisme religieux des citoyens de l'Empire ${ }^{6}$.

Entre ces deux phases se situe un intervalle, correspondant à la période entre la fin de la dynastie attalide et l'instauration du Principat. L'interruption des grands programmes monumentaux, particulièrement évidente quand on pense à la rapidité avec laquelle le paysage civique de Pergame avait été monumentalisé, théâtralisé par un système de terrasses et "marmorisé " sous Attale I, Eumène II et Attale II, se comprend mieux à la lumière de la documentation épigraphique de cette période. Celleci révèle une cité tombée, comme plusieurs autres en Asie Mineure, dans l'incertitude de la guerre d'Andronique et ensuite des guerres de Mithridate et des guerres civiles romaines. Dans ce contexte, l'initiative financière des membres des grandes familles de l'aristocratie constitua souvent la seule opportunité pour maintenir les fêtes civiques et pour entretenir les sanctuaires et les autres espaces publics ${ }^{7}$.

6 Pendant cette époque pleine d'incertitude, la survie du culte pour les souverains attalides témoigne de l'attachement fort de la cité à la mémoire de sa grandeur passée, comme le montre, dans le deuxième quart du $\mathrm{i}^{\mathrm{er}}$ siècle, le soin accordé au culte attalide par Diodoros Pasparos, le grand bienfaiteur pergaménien de la période des guerres de Mithridate ${ }^{8}$. En revanche, le passage au Principat se signale par le remplacement des honneurs cultuels pour la dynastie attalide, peut-être déjà disparus pendant les guerres civiles, avec ceux pour le Princeps ${ }^{9}$. Selon une hypothèse séduisante de Filippo Coarelli, l'introduction du culte impérial à Pergame fut caractérisé par la superposition des honneurs pour Auguste à un lieu symbole de la dynastie: le sanctuaire d'Athéna Niképhoros ${ }^{10}$. Le deuxième temple néocorique, érigé en l'honneur de Trajan, constitue d'ailleurs un exemple tellement abouti de re-fonctionnalisation de l'espace sacré de la période attalide qu'on ignore à présent quel temple ou quelle autre structure précéda le Traianeum sur la terrasse la plus haute de l'acropole ${ }^{11}$. Quant au troisième néocorat, établi sous Caracalla, son identification est sujette à débat et l'hypothèse localisant le temple du culte impérial dans la terrasse du théâtre sera discutée dans la deuxième partie de cette contribution. 
7 À travers les cas d'étude de l'Asklépieion et du temple de la terrasse du théâtre, on analysera plus en détail la documentation textuelle, archéologique et iconographique qui nous permet de saisir les dynamiques et les enjeux du changement du paysage sacré d'une cité grecque dans la longue durée, même dans sa forme plus radicale impliquant l'abandon ou la substitution d'un culte.

\section{Asklépios, les Attalides et le sanctuaire de la vallée du Kaïkos}

8 En 1871, les savants allemands Ernst Curtius et Heinrich Gelzer découvrirent et copièrent, sur une " pierre errante » remployée dans le pavement en face d'une maison dans le village de Kiliseköy (aujourd'hui appelé Zeytindağ), à quelques kilomètres au nord-est de la ville d'Élaïa, un décret de Pergame en faveur d'Attale III, émis à l'occasion du retour triomphal d'une campagne militaire (IvP I $246 ; 138-133$ av. J.-C.) ${ }^{12}$. Parmi les honneurs attribués par le peuple de Pergame à son bienfaiteur, le décret mentionne la consécration d'un agalma du souverain, représenté cuirassé et débout sur le butin de guerre, à l'intérieur du temple d'Asklépios Sôter, de manière à faire du roi le synnaos du dieu ${ }^{13}$. De plus, à l'occasion d'une commémoration annuelle du jour du retour du roi, une procession aura lieu entre le prytanée et le «sanctuaire d'Asklépios Sôter et du roi ", culminant avec un sacrifice et un banquet des magistrats dans ce sanctuaire ${ }^{14}$. Enfin, les dernières lignes du décret stipulent que l'affichage de la stèle devra se faire « dans le sanctuaire d'Asklépios, en face du temple $»^{15}$.

Après l'identification de Pergame comme instance émettrice de l'inscription ${ }^{16}$, le sanctuaire mentionné dans le décret d'Attale III a toujours été identifié avec l'Asklépieion situé en dehors de Pergame, à environ $2 \mathrm{~km}$ à l'ouest des murs d'Eumène II, dans la vallée du Kaïkos. Ce sanctuaire constitue l'un des exemples les plus connus du rayonnement du culte d'Asklépios depuis Épidaure, à partir du IV s. av. J.-C. ${ }^{17}$, ainsi que le cas le plus abouti de l'architecture sacrée de Pergame après son renouvellement datant de la période impériale ${ }^{18}$. Les deux phases de monumentalisation du sanctuaire correspondent à la période attalide, entre le début de la dynastie ${ }^{19}$ et le règne d'Eumène II, et à la haute époque impériale, quand, après une période de déclin, la structure du sanctuaire attalide fut complètement réaménagée, à l'exception du nucléus ancien abritant le temple d'Asklépios ${ }^{20}$.

10 Pour ce qui est de la phase hellénistique, les fouilles allemandes ont mené à l'identification du cœur du sanctuaire en correspondance d'une plateforme rocheuse (Felbsbarre) dans la partie nord-ouest du grand complexe sacré de l'époque romaine (Fig. 2). Cette aire, riche en sources d'eau qui surement contribuèrent à l'implantation du culte d'Asklépios, abrita des activités cultuelles, peut-être de nature non encore institutionnalisée, au moins dès la fin du v viècle, mais ce fut à partir du deuxième quart du III $^{\mathrm{e}}$ siècle (Bauphase 4) que le sanctuaire reçut les premières structures monumentales - temples, autels, un édifice pour l'incubation ${ }^{21}$, des systèmes de canalisation de l'eau des sources destinée aux rituels de purification, un ou plusieurs édifices pour les banquets ${ }^{22}$ - témoignant d'un culte désormais directement lié aux institutions de Pergame ${ }^{23}$. 




Fig. 2. Section hellénistique de l'Asklépieion dans la vallée du Kaïkos. En haut à gauche, la Felsbarre, cœur originaire du sanctuaire ; d'après $A v P X I .2$.

La première phase d'édification du sanctuaire s'insère dans le projet de monumentalisation de Pergame, une citadelle fortifiée destinée, selon le modèle hécatomnide suivi par les premiers dynastes Philétaire et Eumène $\mathrm{I}^{\mathrm{er}}{ }^{24}$, à devenir la capitale d'une dynastie indépendante avec des ambitions régionales. La destruction du sanctuaire par l'armée de Philippe V, en 201 av. J.-C., rendit nécessaire sa reconstruction, qui eut lieu sous Eumène II. À cette époque, l'agrandissement du téménos et la construction de nouveaux édifices fonctionnels à la vie du sanctuaire témoignent de l'intérêt du souverain à donner à l'Asklépieion une monumentalité et une capacité d'accueil à la hauteur d'une capitale exerçant désormais son influence à une échelle à la fois égéenne et anatolienne ${ }^{25}$.

Bien que l'identification précise du temple d'Asklépios parmi les édifices érigés sur la Felsbarre reste problématique ${ }^{26}$, la critique a généralement associé les honneurs pour le roi Attale III dans le décret pergaménien IvP I 246 avec ce sanctuaire hors-les-murs, considéré par plusieurs savants comme le seul Asklépieion qui ait jamais existé à Pergame. Cependant, dès le début $\mathrm{du} \mathrm{xx}^{\mathrm{e}}$ siècle, les tentatives des archéologues allemands d'identifier les destinataires divins de plusieurs édifices sacrés dégagés par les fouilles sur la colline de Pergame, combinées à la discussion de la double iconographie d'Asklépios - trônant ou debout - dans le monnayage pergaménien, ont amené à la proposition qu'un deuxième sanctuaire du dieu ait pu exister dans la cité. Les candidats pour cette identification furent au nombre de deux. Par ordre chronologique, ce fut d'abord le temple ionique de la terrasse du théâtre à être pointé par les experts de la numismatique pergaménienne de la période impériale ${ }^{27}$. Cependant, cette hypothèse n'a jamais obtenu le consensus des archéologues de l'école allemande, qui, depuis l'étude fondamentale de Hans von Prott sur le culte de Dionysos 
Kathêgémôn chez les Attalides, ont toujours attribué le temple de la Theaterterrasse à ce dieu $^{28}$. Le dossier concernant ce temple a été repris récemment par l'archéologue et historien de l'art Filippo Coarelli, dans le cadre d'une analyse ambitieuse du paysage sacré de Pergame que l'on pourrait qualifier d'une «anti-summa» de l'interprétation offerte par l'école allemande, et qui a attribué au décret IvP I 246 pour Attale III une place nouvelle dans la discussion du dossier de la Theaterterrasse : si le décret avait été utilisé auparavant comme la preuve de l'existence d'un seul Asklépieion à Pergame ${ }^{29}$, selon Coarelli les indications fournies par ce texte renforceraient les indices numismatiques et archéologiques dans la direction de l'identification du temple du théâtre comme un Asklépieion, et précisément celui où se situeraient les activités rituelles mentionnées dans le décret.

Avant de nous consacrer à une évaluation de cette hypothèse, il convient d'examiner le deuxième candidat avancé par la critique pour l'identification du deuxième Asklépieion de Pergame : le temple R, occupant la petite terrasse sur le versant sud de la colline, entre le gymnase d'Eumène II à l'est, les thermes romains au sud-ouest, l'édifice à podium $\mathrm{H}$ au nord, et le sanctuaire de Déméter plus loin au nord-ouest. Le dossier épigraphique et archéologique $\mathrm{du}$ temple $\mathrm{R}$ a été exploré en détail par l'école allemande, avec des résultats qui invitent à rejeter l'hypothèse d'une identification avec un lieu de culte d'Asklépios. Il sera néanmoins utile de reprendre brièvement ce dossier car les indications méthodologiques que l'on peut en tirer nous aideront dans le réexamen de la documentation concernant le temple de la terrasse du théâtre ${ }^{30}$.

\section{Un deuxième Asklépieion à Pergame ? L'hypothèse du temple $\mathrm{R}$ du gymnase}

15 L'édifice R est un petit temple tétrastyle d'ordre ionique, situé sur une terrasse qui s'élève environ $10 \mathrm{~m}$ au-dessus de celle du gymnase. Une particularité de ce temple est qu'il est composé d'éléments architecturaux appartenant à l'origine à un temple plus grand, d'ordre dorique ${ }^{31}$. Dans le cadre d'une réinterprétation générale de la documentation concernant la figure de l'artiste pergaménien Phyromachos ${ }^{32}$, l'archéologue Bernard Andreae édita en 1990 un dossier de contributions visant à expliquer l'anomalie du temple $\mathrm{R}$ comme le résultat du transfert et de la reconstruction, à une échelle mineure et avec une décoration ionique, d'un temple dorique situé originairement dans un sanctuaire de la vallée du Kaïkos : ce temple aurait été le Niképhorion, détruit en 156/5 av. J.-C. par l'armée du roi Prousias II de Bithynie $^{33}$. On sait depuis Polybe et Diodore que, lors de son incursion, Prousias vola une statue cultuelle fameuse d'Asklépios, réalisée par Phyromachos ${ }^{34}$. Andreae combina ces données avec un dossier d'inscriptions et de statuettes provenant du temple $\mathrm{R}$ et de ses environs, qui avait déjà suggéré à Erwin Ohlemutz, dans son étude des cultes et sanctuaires de Pergame, l'hypothèse que le temple $\mathrm{R}$ ait pu abriter un culte d'Asklépios ${ }^{35}$.

Il n'est pas nécessaire de revenir en détail sur cette hypothèse, car l'argumentation d'Andreae a été démontée pièce par pièce dans une contribution magistrale de Helmut Müller en $1992^{36}$. Comme on l'a dit ci-dessus, il n'est cependant pas sans intérêt de résumer les raisons méthodologiques pour lesquelles l'identification du temple $\mathrm{R}$ comme un Asklépieion ne peut être retenue. Une interprétation erronée du contenu et de l'encadrement chronologique de la documentation épigraphique concernant le 
temple $\mathrm{R}^{37}$ a été supportée par l'identification douteuse d'un grand torso retrouvé dans la salle $\mathrm{X}$ des thermes romains du gymnase, en bas du temple, avec une statue cultuelle d'Asklépios provenant du temple ${ }^{38}$. Enfin, le dossier a été renforcé par l'attribution hypothétique, et parfois forcée, à ce seul édifice cultuel d'un certain nombre de statuettes d'Asklépios, appartenant à des périodes différentes et associées à des contextes de découverte topographiquement variés (là où ceux-ci sont connus), et parfois même à des espaces domestiques ${ }^{39}$.

17 Aux observations décisives de Müller, il convient d'ajouter que, selon la critique plus récente, la double phase de décoration des pièces architecturales ne témoigne pas de leur transfert depuis un autre sanctuaire de Pergame : elle renvoie plutôt à une phase de réédification d'un temple antérieur daté, sur la base du style, de la seconde moitié $\mathrm{du}$ III $^{\mathrm{e}}$ siècle ${ }^{40}$, suite à la réorganisation de l'aire du gymnase pendant le règne d'Eumène II. L'agrandissement du gymnase doit en effet avoir impliqué le réaménagement de la terrasse sur laquelle le prédécesseur du temple $R$ avait été construit $^{41}$. L'histoire du temple $\mathrm{R}$ s'insère donc dans la même phase de réaménagement du paysage sacré de Pergame que l'agrandissement de l'Asklépieion dans la vallée, et probablement, comme on le verra par la suite, de l'érection du temple ionique de la terrasse du théâtre. Cette phase se caractérise par le succès de l'ordre ionique, qui vient remplacer l'ordre dorique employé dans les temples érigés sous les premiers dynastes ${ }^{42}$.

Pour revenir à l'hypothèse d'Andreae, on peut conclure que la datation du temple ionique $\mathrm{R}$ au règne d'Eumène II rend infondée la théorie selon laquelle la construction de cet édifice aurait suivi l'incursion de Prousias II, pendant les premières années de règne d'Attale II. Dans la section suivante, on reviendra au dossier de la Theaterterrasse. Dans les différentes sous-sections, on examinera successivement la documentation archéologique, numismatique, sculpturale et épigraphique, pour mettre à l'épreuve l'hypothèse que ce temple ait hébergé un culte d'Asklépios. Suivant les précautions auxquelles nous invite le dossier du temple $\mathrm{R}$, on prêtera une attention particulière à la chronologie de la documentation ainsi qu'à sa distribution topographique.

\section{Le temple ionique de la Theaterterrasse}

Un temple ionique tétrastyle $(21,60 \times 13,17 \mathrm{~m})$, situé sur un haut podium $(4,5 \mathrm{~m}$ de hauteur) qui en fait un point de repère majeur dans le profil de Pergame, occupa, probablement à partir du début du $\mathrm{II}^{\mathrm{e}}$ siècle av. J.-C., l'extrémité nord de la terrasse du théâtre, une plateforme longue et étroite positionnée au milieu du versant occidental de la citadelle et délimitée à l'ouest par une stoa, à l'est par l'entrée du théâtre et au sud par les pentes de la terrasse supérieure de l'agora ${ }^{43}$. Aucun indice matériel ou textuel de la période hellénistique ne nous permet d'associer le temple avec une divinité précise ${ }^{44}$. Pourtant, comme on a anticipé ci-dessus, sa proximité avec le théâtre a suggéré un lien avec Dionysos Kathêgémôn.

Comme les études archéologiques plus récentes l'ont montré, l'érection du temple constitua la dernière étape dans l'aménagement de l'aire sacrée, à dater probablement entre la fin du règne $d^{\prime}$ 'Attale $I^{\text {er }}$ et celui d'Eumène II, bien qu'une date sous les derniers Attalides ne puisse être exclue ${ }^{45}$. Dans cette perspective on peut apprécier la différence d'orientation de $90^{\circ}$ entre l'autel, antérieur au temple et orienté à l'est, et le temple luimême, suivant une orientation nord-sud ${ }^{46}$. Les parties ouest, nord et est du temple appartiennent à la période hellénistique, tandis que la partie frontale remonte à la 
phase de restauration romaine. Des traces d'incendie furent dégagées par les fouilleurs, ce qui a permis d'expliquer que le temple fut partiellementdétruit, peut-être temporairement abandonné, et ensuite reconstruit pendant l'époque impériale. La structure préservée de l'autel date aussi de cette nouvelle phase d'utilisation du temple ${ }^{47}$.

La date de reconstruction du temple demeure incertaine. Les fragments de l'épistyle du temple romain ont préservé les traces des pivots utilisés pour fixer au marbre les lettres en bronze doré d'une dédicace concernant un empereur. Se fondant sur la disposition des trous d'encrage, Fabricius et Bormann arrivèrent à restituer la première ligne du texte, publié par Max Fränkel dans IvP II 299 et ensuite amélioré par Barbara Burrel dans son étude des cultes impériaux dans les cités d'Asie Mineure. On reporte ici le texte après la révision de Burrel ${ }^{48}$ :

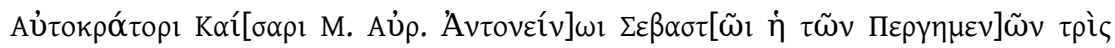

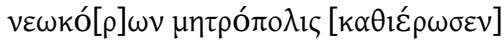

Se fondant sur une comparaison entre la différente disposition des pivots pour $O$ et $\Omega$, Fränkel accepta la restitution d'une dédicace faite à un empereur, au datif, plutôt que

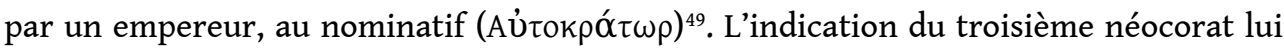
suggéra d'identifier l'empereur mentionné avec Caracalla, puisqu'on sait que ce fut sous cet empereur, en 213/4 ap.J.-C., que Pergame reçut son troisième culte néocorique ${ }^{50}$. Cela permettrait de dater la reconstruction du temple du règne de Caracalla. Cependant, en revenant sur le dossier architectonique, Strocka a rejeté les implications chronologiques de cette lecture de l'inscription à l'appui d'une analyse stylistique des éléments décoratifs du temple, qui présentent plusieurs traits communs avec ceux du deuxième temple néocorique, le Traianeum. Ces considérations suggèrent une date sous Hadrien pour la reconstruction du temple ${ }^{51}$. Récemment, la contradiction apparente entre le contenu de l'inscription et les données archéologiques a été résolue par Richard Posamentir, qui, dans le cadre d'une étude systématique des inscriptions en lettres de bronze dorées de l'Asie Mineure impériale, a proposé une nouvelle lecture de la dédicace sur l'épistyle du temple ionique de Pergame :

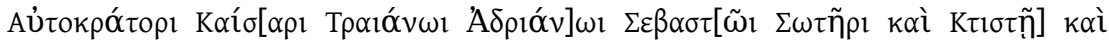

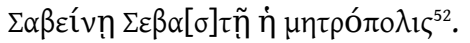

La disparition de l'indication du troisième néocorat et surtout son remplacement par le nom de l'impératrice Sabine imposent la restitution du nom d'Hadrien dans la lacune. L'analyse croisée du vocabulaire de la dédicace et des sources historiques suggère à Posamentir de dater le texte entre 124 et 129 ap. J.-C., peut-être à l'occasion d'une visite

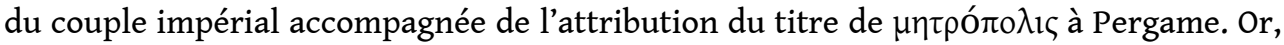
notre dédicace ainsi datée soulève une question fondamentale à propos de la relation entre la consécration du temple restauré au couple impérial et la lettre de 137 par laquelle Hadrien refusa la proposition de la cité de Pergame de consacrer un troisième temple néocorique après ceux d'Auguste et de Trajan, situés au sommet de l'acropole ${ }^{53}$. Comme l'observe Posamentir, la lettre d'Hadrien ne contient aucune mention de la dédicace du temple de la Theaterterrasse. Pourtant, on peut observer que ce temple, déjà consacré au couple impérial, aurait constitué un candidat idéal pour le projet d'instituer un nouveau culte néocorique au sein de l'ancienne cité, bien qu'un autre endroit, peut-être dans l'Asklépieion, aurait pu lui aussi offrir une solution convenable à la mise en valeur du nouveau culte ${ }^{54}$. Quoiqu'il en soit, la lettre d'Hadrien eut comme conséquence de changer les projets des Pergaméniens, qui suivirent la proposition de 
l'empereur d'ériger son portrait à l'intérieur du temple de Trajan. Les fouilles du Traianeum ont dégagé les restes de cette statue colossale d'Hadrien, située à côté de celles de Zeus Philios et de Trajan ${ }^{55}$. Près de la statue fut affichée la grande plaque en marbre avec la lettre de l'empereur, destinée à légitimer l'initiative de la cité et à lui assurer au moins une partie du prestige auquel elle visait par la tentative inaboutie d'établir un troisième néocorat.

\section{La documentation numismatique de la période impériale} l'institution du troisième néocorat de la cité témoignent de la double iconographie d'une divinité que l'attribut d'un serpent dans la main permet d'identifier avec Asklépios. La statue cultuelle représente le dieu soit debout, soit assis sur un trône, dans un temple hexastyle ou tétrastyle ${ }^{56}$. Avant que la thèse de von Prott identifiant le temple de la Theaterterrasse avec un lieu de culte de Dionysos ne s'impose dans la vulgate archéologique, ces monnaies avaient convaincu Hans von Fritze de l'existence, à Pergame, de deux Asklépieia caractérisés par une iconographie différente de la statue cultuelle ${ }^{57}$. Bien que l'hypothèse d'un deuxième temple d'Asklépios à l'intérieur des murs n'ait guère convaincu ${ }^{58}$, elle fut reprise par Erwin Ohlemut ${ }^{59}$ et, plus récemment, par Simon Price dans son répertoire des sanctuaires du culte impériale en Asie Mineure $^{60}$.

Coarelli a récemment relancé cette interprétation en distinguant entre le dieu debout, généralement accepté comme le type de la statue cultuelle de l'Asklépieion hors-lesmurs, et le dieu assis, à identifier avec la statue du temple de la Theaterterrasse ${ }^{61}$. Une partie des monnaies émises pour Caracalla associe la statue au dieu debout avec la vision frontale d'un temple seul, identifié avec l'ancien temple sur la Felsbarre ${ }^{62}$. À cette image s'oppose, dans d'autres types monétaires, la représentation du dieu sur un trône, à l'intérieur d'un temple seul ${ }^{63}$, ou entouré de deux autres temples. Sur les monnaies appartenant à ce dernier type (Fig. 3), des sigles souvent associés aux trois édifices permettent d'identifier les destinataires des trois cultes néocoriques: Auguste (АҮГ), Trajan (TP) et Caracalla (AN). Selon l'identification proposée par Coarelli, qui attribue cet édifice à Caracalla en suivant le texte de l'épistyle du temple de la Theaterterrasse publié dans IVP II 299, cette composition triadique évoquerait les trois sanctuaires néocoriques sur l'acropole de Pergame. Les trois temples du culte impérial seraient ainsi visibles, l'un près de l'autre, au sommet de la partie nord-ouest de la colline, bien que sur des terrasses différentes : le plus ancien, dédié à Rome et Auguste (localisé par Coarelli dans le temple d'Athéna), le deuxième pour Trajan et Zeus Philios (le Traianeum, dans la terrasse occupant la partie nord-ouest de la citadelle) et le dernier, qui serait précisément le temple ionique de la Theaterterrasse ${ }^{64}$. La solution iconographique des trois temples juxtaposés aurait dès lors la fonction symbolique de souligner la continuité entre les trois néocorats de Pergame et la fonction topographique de restituer virtuellement leur proximité au sommet de l'acropole. 




Fig. 3. Bronze de Pergame représentant les trois temples du culte impérial (cf. BuRRELl [2004], p. 31, type 23 - Wikimedia Commons).

Pour fascinante qu'elle soit, cette reconstruction nécessite d'être reconsidérée à la lumière de plusieurs détails qui nous obligent à la nuancer. Tout d'abord, si on voulait retenir l'identification du temple de la Theaterterrasse avec celui consacré au culte de Caracalla malgré la nouvelle lecture du texte de l'épistyle proposée par Posamentir, nous serions forcés de postuler une nouvelle consécration du temple en $213 / 4$, qui pourtant n'aurait pas effacé la dédicace précédente pour Hadrien. De plus, la proximité entre les trois temples néocoriques dans l'iconographie numismatique ne reflète pas nécessairement une véritable proximité topographique ${ }^{65}$. Un troisième point à discuter concerne l'iconographie d'Asklépios à Pergame. À ce propos, il est utile de rappeler l'étude de Kranz qui, parmi d'autres, a beaucoup nuancé l'idée qu'il existait une continuité claire, dont témoigneraient les monnaies de Pergame, entre la statue cultuelle de l'Asklépios d'Épidaure et la figure du dieu assis sur les (rares) monnaies hellénistique représentant le dieu assis sur un trône. Des différences significatives interviennent aussi entre les monnaies hellénistiques de Pergame au dieu assis et celles de la période impériale ${ }^{66}$. Il semblerait même que l'iconographie monétaire de la période romaine soit plus directement inspirée du modèle d'Épidaure qu'on puisse le dire pour l'iconographie des monnaies hellénistiques. De plus, le manque presque total d'attestations de l'iconographie du dieu assis dans la documentation non monétaire de la Pergame hellénistique invite à prendre des distances avec l'hypothèse qu'une statue cultuelle d'Asklépios trônant ait joué un rôle important dans la caractérisation du dieu à l'époque de la dynastie attalide. On ajoutera enfin que c'est seulement à partir du règne d'Antonin le Pieux que l'on connait à Pergame des émissions représentant à nouveau Asklépios trônant ${ }^{67}$. En conclusion, il n'est pas possible d'établir une opposition binaire durable entre le type d'Asklépios débout et le sanctuaire extra muros d'une part, et d'autre part, entre le dieu trônant et un sanctuaire localisé à l'intérieur 
de la ville. Les deux figures ont des histoires distinctes, dont les étapes ne peuvent être généralisées à toute l'histoire de Pergame hellénistique et impériale.

Enfin, les arguments apportés par Coarelli en faveur de l'identification du temple avec un lieu de culte d'Asklépios plutôt que de Dionysos ne sont pas conclusifs. En effet, d'un côté, le savant italien a raison de faire observer que la dédicace d'un portail à Dionysos Kathêgémôn en regard de l'entrée nord de la scène du théâtre, à la période hellénistique tardive, n'implique pas, en soi, que le temple placé dans les alentours fût consacré à Dionysos, car le théâtre était lui-même un lieu lié à ce dieu et le centre de l'activité des Technites de Dionysos ${ }^{68}$. L'identification du dieu vénéré dans le temple avec Dionysos se fonderait dès lors, selon Coarelli, sur la seule hypothèse que ce dieu conviendrait bien à un temple situé près du théâtre ${ }^{69}$ En revanche, la thèse de l'identification du temple ionique avec un Asklépieon est soutenue par Coarelli qui invoque deux pièces trouvées dans l'aire du temple : un petit autel dédié à Asklépios et Hygieia, découvert près du coin sud-ouest du temple ionique; le visage d'une statue plus grande que nature d'un Attalide, dégagé à l'intérieur de la cella et qui, selon Coarelli, correspondrait à l'agalma d'Attale III dont la consécration dans le temple d'Asklépios est mentionnée dans le décret IvP I 246.

J'examinerai ces deux objets dans la section suivante, pour montrer qu'ils n'offrent pas d'indices probants de la présence d'un culte d'Asklépios sur la Theaterterrasse à l'époque hellénistique.

\section{Un autel portatif d'Asklépios Sôter et Hygieia et un visage d'Attale III}

Le petit autel en marbre IvP II 312, dédié à Asklépios Sôter et Hygieia $(19 \times 25 \times 12 \mathrm{~cm}$; Fig. 4a-b) présente deux particularités : à la base du fût se trouve une décoration à méandres inachevée; le texte de la dédicace appartient à deux mains, sinon à deux périodes différentes. 


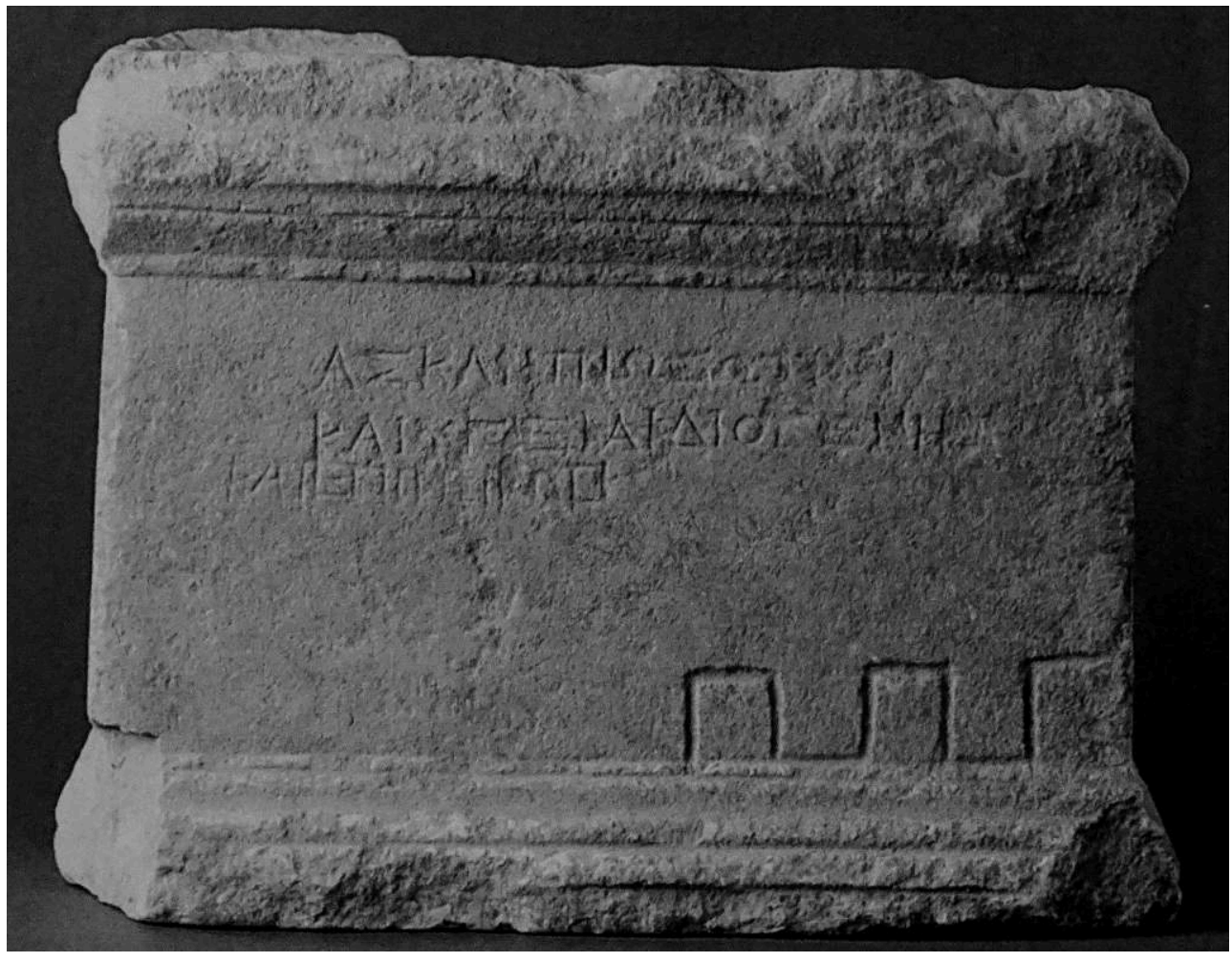

Fig. 4a. L'autel pour Asklépios Sotêr et Hygieia IVP I| 312, d'après GRüßINGER - KÄSTNER - SCHOLL (2011), p. $544, n^{\circ} 6.36$



Fig. 4b. Fac-similé de l'autel, d'après IVP II 312.

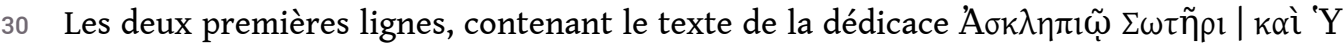

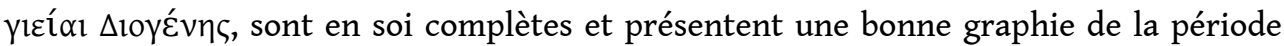
républicaine ou du début de l'empire, cohérente avec le détail de l'omission du iota dans le datif du nom du dieu. En revanche, la troisième ligne, inachevée, contient le 


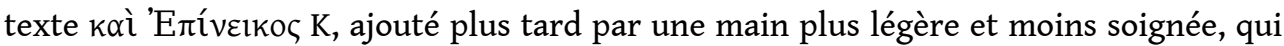
n'a pas respecté la mise en forme de l'inscription précédente en ce qui concerne l'alignement à gauche comme l'espace d'interligne. Cette nouvelle phase de l'inscription montre l'utilisation d'un sigma lunaire carré, documenté à Pergame dans des textes peu soignés de la période romaine ${ }^{70}$. Le lapicide peu compétent abandonna son travail après avoir gravé un $\mathrm{K}$, interprété par l'éditeur, Max Fränkel, comme la

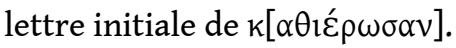

31 Comment interpréter cet artefact, modeste, déplaçable et témoignant d'une initiative privée, par rapport à son lieu de découverte ? Coarelli paraît surestimer son rôle - qui demeure d'ailleurs isolé - dans la caractérisation du temple comme lieu de culte d'Asklépios. Il considère que cet objet témoigne de la phase attalide, alors que, selon toute vraisemblance, ce n'est pas le cas, et il exclut son déplacement de l'Asklépieion hors-les-murs jusqu'à la terrasse du théâtre. De fait, ses petites dimensions rendraient cette hypothèse tout à fait possible, d'autant plus que l'analyse de l'inscription a suggéré une double phase d'utilisation, pour laquelle on ne connait ni le propos précis, ni la raison de l'abandon de la pierre avant l'achèvement de la troisième ligne. D'ailleurs, même en acceptant que l'objet ait été retrouvé dans son lieu de dédicace originaire, il faudra rappeler ce qu'on a dit à propos du dossier du temple $\mathrm{R} d \mathrm{du}$ gymnase : faute d'indices ultérieurs et plus solides, la présence, dans plusieurs lieux de Pergame, de dédicaces privées à une divinité aussi importante pour la cité qu'Asklépios ne peut être acceptée comme une preuve suffisante de l'existence de plusieurs lieux de cultes officiels pour cette divinité.

Venons-en maintenant au visage en marbre d'un Attalide, trouvé dans la partie nord de la cella du temple ionique, près du mur de fond, et à l'hypothèse de Coarelli selon laquelle ce fragment appartiendrait à l'agalma d'Attale III mentionné dans IvP I 246. L'analyse stylistique proposée par Coarelli situe de manière convaincante la statue dans la deuxième moitié du II $^{\mathrm{e}}$ siècle, donc sous Attale II ou III ${ }^{71}$. Selon l'auteur, la mesure de la tête $(25 \mathrm{~cm}$, dont $22,5 \mathrm{~cm}$ pour le visage) serait parfaitement cohérente avec l'indication d'IvP I 246, ligne 7, qui identifie la hauteur de la statue d'Attale à 5 coudées $^{72}$. La surface postérieure du portrait montre les traces d'un pivot en métal utilisé pour attacher le visage à un support.

En commentant la mesure de la base (d'époque impériale) trouvée dans la cella du temple, qui est assez large pour héberger deux statues, Coarelli propose que la statue du roi, montée sur le butin de guerre, serait représentée à côté de celle d'Asklépios assis, et que l'introduction du culte de Caracalla dans le temple aurait été accompagnée de la substitution du visage du roi hellénistique par celui de l'empereur. À cet égard, Coarelli propose que la tête d'Attale III fut sciée pour accueillir un nouveau visage et pour permettre ainsi un changement demandant une intervention très limitée ${ }^{73}$.

Cette hypothèse se heurte à plusieurs oppositions. Pour commencer, si le culte des Attalides a surement survécu à la fin de la dynastie, comme on l'a vu, rien ne suggère toutefois qu'il soit resté en fonction encore à la période impériale. Au contraire, la stratigraphie de la découverte du portrait d'Attale III dans le temple de la Theaterterrasse impose de manière décisive une réponse négative ${ }^{74}$. Deuxièmement, le détail de la substitution du visage d'Attale III, détaché du reste de la statue et abandonné pour faire place à celui d'un empereur, est erroné et superflu, car la présence d'un mécanisme pour accrocher le visage à un support s'explique de manière plus convaincante comme une solution commune pour une statue cultuelle acrolithe, à 
savoir une statue composée d'une armature en bois couverte de vêtements et attachée à des extrémités en marbre représentant les membres et le visage. Ceci fut sans doute le cas pour plusieurs statues cultuelles de souverains hellénistique et les Attalides ne font pas exception à cet égard ${ }^{75}$.

Une troisième considération, plus générale, concerne la grande richesse de la statuaire royale pergaménienne de la période attalide. En particulier pendant la phase mature de l'histoire de la dynastie, au milieu du $\mathrm{II}^{\mathrm{e}}$ siècle, on assiste à une multiplication de portraits et de statues cultuelles des membres de la maison royale. Cette tendance était destinée à se poursuivre lorsque Pergame fut intégrée à la province romaine d'Asie, comme le suggère le dossier du bienfaiteur Diodoros Pasparos, responsable, pendant le deuxième quart du $\mathrm{i}^{\mathrm{er}}$ siècle, de la restauration d'un groupe sculptural de la dynastie situé dans le gymnase de la cité ${ }^{76}$. Cette abondance nous suggère que pratiquement chaque édifice public aurait pu héberger un ou plusieurs portraits de membres de la famille royale ${ }^{77}$. Par conséquent, l'identification du visage d'Attale III dans le temple de la Theaterterrasse avec l'agalma mentionné dans le décret IvP I 246 demeure une hypothèse non prouvée. À la lumière de la discussion suivante, il parait en effet bien plus probable que le fragment de cette statue acrolithe et le décret ne représentent que deux cas parallèles et distincts d'une pratique honorifique commune à Pergame.

Par souci d'exhaustivité, je voudrais aussi attirer l'attention sur un aspect accessoire de l'argumentation de Coarelli qui est sans doute à rejeter. Il s'agit de l'identification du temple de la Theaterterrasse avec l'Attaleion mentionné dans la documentation de l'association cultuelle des Attalistai de Téos, fondée par Kratôn de Chalcédon, flûtiste et membre bienfaiteur des Technites de Dionysos ${ }^{78}$. Cette identification se heurte à deux observations contraires. La première concerne le fait que, puisque toute la documentation de cette association a été repérée à Téos, la localisation de leur Attaleion dans cette cité demeure plus probable qu'à Pergame même ${ }^{79}$. La deuxième - et elle est décisive - est que le téménos des Attalistai était une propriété privée du fondateur Kratôn, qui la consacra aux usages cultuels de l'association pendant sa vie et en transféra ensuite la propriété à ses confrères dans son testament ${ }^{80}$. L'étude des lieux de réunion privés de ce type de congrégations cultuelles (et souvent professionnelles) met en valeur un type de structure combinant souvent une salle de banquet avec d'autres pièces destinées à des fins cultuelles et/ou de stockage ${ }^{81}$. Par conséquent, si on acceptait une localisation pergaménienne du téménos des Attalistai, l'ancienne identification avec le Nischenbau ${ }^{82}$, situé à l'extrémité sud de la terrasse du théâtre, offrirait encore une localisation plus probable que le temple ionique. Dans tous les cas, lorsqu'une association privée se réunit dans un sanctuaire existant, elle laisse d'habitude des traces épigraphiques de son passage, ce qui n'est pas le cas pour les Attalistai dans l'aire du temple ionique en particulier, et à Pergame en général. En conclusion, l'attribution aux Attalistai d'un espace cultuel qui lui serait propre dans la terrasse du théâtre semble devoir être rejetée.

\section{Les lieux d'affichage des documents mentionnant l'Asklépieion de Pergame}

En laissant de côté pour l'instant le problème du dieu vénéré dans le temple de la Theaterterrasse, cette section sera consacrée à une analyse de la correspondance entre les lieux d'affichage évoqués par trois documents pergaméniens des III $^{\mathrm{e}}$ et II ${ }^{\mathrm{e}}$ siècle av. J.- 
C. et les sites où les stèles ont été effectivement trouvées. Notre documentation comprend trois inscriptions pergaméniennes - un accord entre le roi et ses mercenaires et deux décrets - qui stipulent l'affichage du texte dans l'Asklépieion de Pergame.

Le document le plus ancien est une stèle (IvP I $13 ; 263-241$ av. J.-C.) contenant le texte d'un accord entre Eumène $\mathrm{I}^{\text {er }}$ et ses mercenaires en garnison dans les forteresses de Philétaireia (aux pieds de l'Ida) et d'Attaleia (près de Thyateira) ${ }^{83}$. L'inscription mentionne quatre localités où le texte sera exposé, dont l'une "à Pergame, dans le sanctuaire d'Athéna ${ }^{84} »$. Selon l'indication de l'éditeur, la stèle a été recomposée à partir de deux morceaux, trouvés tous les deux en 1883 : l'un, plus grand, avait été réutilisé dans la cour d'une maison privée érigée sur le versant sud-ouest de la colline de Pergame, près du gymnase (jadis le quartier grec de Gurnellia, aujourd'hui nommé Büyük Alan); l'autre fut dégagé dans les ruines des murs byzantins érigés au sud-ouest de l'agora. Puisque le décret avait été affiché plus haut, dans la terrasse d'Athéna, et que les deux fragments ont été retrouvés dans deux lieux différents, mais situés dans la partie sud de la colline, entre la citadelle et la cité basse, on peut avancer l'hypothèse que la stèle fut originairement remployée in toto dans les fortifications byzantines, et que le fragment plus grand fut ensuite déplacé, après leur ruine, et réutilisé ultérieurement un peu plus bas, dans la maison privée que l'on a mentionnée.

Le deuxième texte, datant des années suivant l'extinction de la dynastie, est un décret réglant la prêtrise héréditaire d'Asklépios, attribuée à Asklépiadès et à ses descendants $\left(\right.$ CGRN 206) ${ }^{85}$. Dans ce cas, deux des trois localités choisies pour l'érection des stèles sont des sanctuaires pergaméniens: «le sanctuaire d'Asklépios à Pergame» et «le sanctuaire d'Athéna sur l'acropole $»^{86}$. Les deux fragments préservés de la stèle ont été dégagés par les archéologues allemands respectivement dans la tour carrée placée à l'angle sud-ouest de la terrasse d'Athéna et dans la partie centrale de la Theaterterrasse, à la latitude du Grand Autel. La localisation de la deuxième partie fait penser qu'elle a pu dégringoler de l'angle sud-ouest de la terrasse d'Athéna, le plus proche du temple et lieu privilégié pour la publication de documents officiels pendant la période royale ${ }^{87}$. Il est donc sûr que l'exemplaire préservé du décret concernant la prêtrise d'Asklépios est celui du sanctuaire d'Athéna.

Le troisième document est la pierre errante de Kiliseköy, contenant le décret en l'honneur d'Attale III de retour de sa campagne militaire victorieuse (IvP I 246). Il faut maintenant revenir brièvement sur la question du parcours qui mena cette stèle de Pergame jusqu'à Kiliseköy, à une heure de marche au nord-est de la cité côtière d'Élaïa. Comme l'a montré Robert, la plaine du Kaïkos en face de Pergame, où se trouvait le grand Asklépieion hors-les-murs, a fait l'objet, depuis l'antiquité tardive jusqu'au début des fouilles scientifiques $\mathrm{du} \mathrm{xIX}^{\mathrm{e}}$ siècle, d'une riche histoire de déplacements d'inscriptions, que la plaine ouverte rendait facile à amener à l'ouest, vers la bouche du Kaïkos dans la région d'Élaïa ${ }^{88}$. Il est intéressant de noter que les pierres pergaméniennes retrouvées en bas dans la vallée du Kaïkos proviennent toutes des parties les plus exposées de la cité antique, à savoir le sanctuaire d'Asklépios dans la vallée et le grand gymnase, situés à l'extérieur des fortifications qui, d'abord à l'époque byzantine, puis turque, furent élevées pour protéger la citadelle au sommet de la colline de l'ancienne Pergame (Fig.5). En revanche, les inscriptions originairement exposées dans la cité haute ont été retrouvées pendant les fouilles sous le terrain, ou réutilisées dans les fortifications médiévales, mais de toute façon à une distance limitée 
de leur site originel ${ }^{89}$. Comme on l'a vu plus haut, cette hypothèse est valable aussi pour le fragment de la stèle contenant l'accord d'Eumène ${ }^{\mathrm{er}}$, remployée dans une maison située sur le versant de la colline, à l'intérieur du circuit des murs anciens.

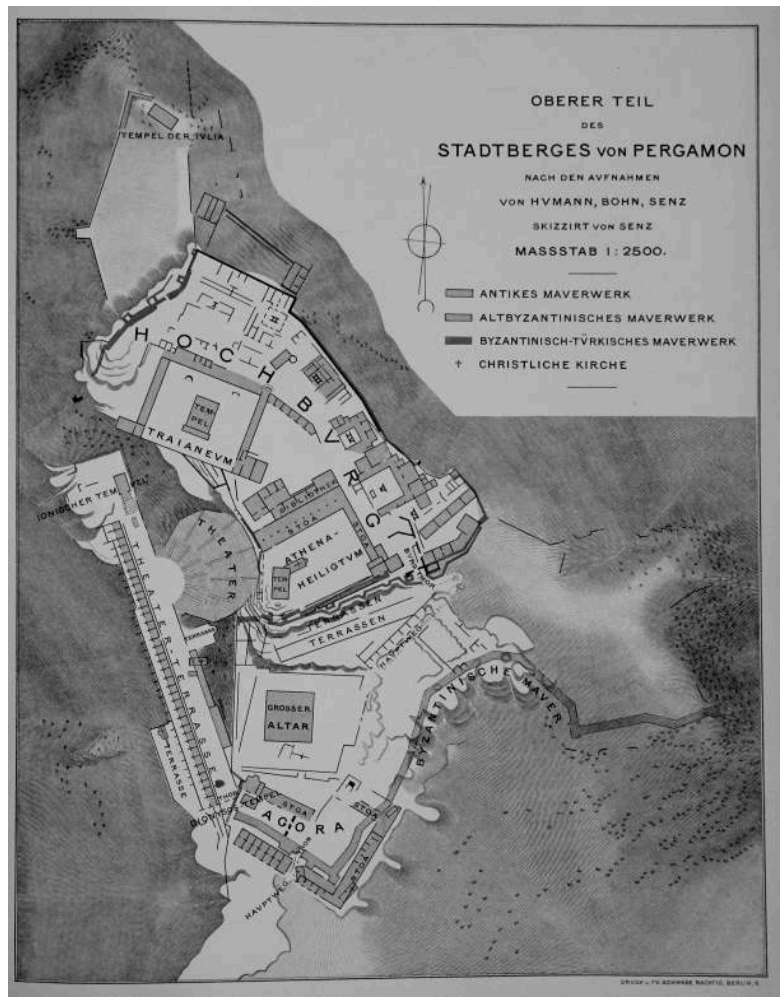

Fig. 5. Plan de la citadelle de Pergame avec les fortifications byzantines et turques, d'après IVPI.

\section{L'unicité de l'Asklépieion dans les inscriptions hellénistiques}

D'autres arguments, concernant le contenu des deux décrets pergaméniens discutés dans la section précédente, confirment la thèse que, pendant les phases attalide et républicaine, il n'y avait à Pergame qu'un seul Asklépieion, et que celui-ci était horsles-murs. Dans le décret concernant le culte d'Asklépios, Asklépiadès est en charge de "la prêtrise d'Asklépios et de toutes les divinités installées dans l'Asklépieion ${ }^{90}$. Cette indication suggère que l'Asklépieion était une structure suffisamment articulée pour accueillir des autels et/ou des statues d'autres dieux, ce qui correspond très bien, on l'a vu, à la topographie du sanctuaire extra-urbain sur la Felsbarre. En revanche, cette caractéristique nous permet d'exclure l'identification d'un éventuel Asklépieion urbain dans le temple de la Theaterterrasse, dont le plan ne laisse évidemment pas de place pour imaginer un ensemble de structures cultuelles agrégées dans un seul sanctuaire ${ }^{91}$. Il en va de même pour la référence, dans le décret en l'honneur d'Attale III, à la célébration d'un banquet des magistrats dans le sanctuaire, en conclusion de la procession annuelle pour l'anniversaire du retour du roi ${ }^{92}$. Encore une fois, on ne voit pas comment un Asklépieion localisé dans le temple de la Theaterterrasse pourrait avoir hébergé un banquet, en considération de l'absence de toute structure adaptée à l'occasion ${ }^{93}$. En revanche, la présence d'une salle de banquet dans le Nordostbau de l'Asklépieion hors-les-murs remonte aux premières phases de la monumentalisation du 
sanctuaire (Bauphase 5 ; milieu du III $^{e}$ siècle); sous le règne d'Eumène II (Bauphase 10 ; premier quart du siècle), la Osthöfe vint élargir de manière considérable les structures du sanctuaire adaptées à l'accueil des fêtes liées au culte d'Asklépios et, à partir d'un certain moment de son règne, celui des honneurs cultuels pour Attale III.

Dans le décret pour Attale, il est question des revenus de l'Asklépieion, à utiliser pour financer la cérémonie annuelle de commémoration du retour $\mathrm{du}$ roi ${ }^{94}$. Cette formule montre que, pour les institutions de Pergame, il n'y avait qu'un lieu de culte civique d'Asklépios. De surcroît, dans le texte concernant la prêtrise du dieu, l'expression ćv


comme l'indication qu'il y avait, dans le territoire de Pergame, deux sanctuaires du dieu, l'un dans la polis et l'autre dans sa chōra $a^{95}$. De manière plus simple et convaincante, la précision apportée par le décret est à entendre par opposition au troisième temple choisi pour l'affichage du texte: l'Asklépieion de Mytilène (lignes 40-41). Enfin, que l'Asklépieion en question ne puisse être que celui hors-les-murs est confirmé par le fait que les inscriptions honorifiques pour les prêtres du dieu, à savoir les descendants de l'Asklépiadès auquel la cité attribua la prêtrise héréditaire, étaient affichées à l'intérieur du sanctuaire de la vallée du Kaïkos ${ }^{96}$.

\section{La procession d'accueil d'Attale III dans IVP I 246}

Pour conclure cet excursus sur l'Asklépieion hors-les-murs à la période hellénistique, on peut maintenant revenir sur la topographie des honneurs décrétés à Attale III dans le texte IvP I 246. On l'a vu, le décret distingue la cérémonie d'accueil (apantésis) du roi, qui eut lieu à l'occasion de son retour d'une campagne militaire victorieuse (lignes 2651), d'une procession annuelle établie après cet évènement, et destinée à le commémorer (lignes 6-26). Dans le texte affiché, cependant, ces deux évènements apparaissent dans un ordre chronologique inverse : cette particularité, qui a longtemps trompé les commentateurs du document, est en effet due à la composition du décret, qui synthétise deux phases de délibération de la part des institutions de Pergame ${ }^{97}$. Quand la nouvelle du retour imminent du roi arriva dans la capitale, la cité décréta une série d'honneurs concernant la cérémonie d'accueil. Cette section n'était plus à l'ordre du jour au moment où, après la fête, le peuple de Pergame décida d'enrichir le dossier des honneurs pour Attale III avec d'autres initiatives, pensées pour commémorer la victoire et les bienfaits du roi à jamais. C'est justement le contenu de cette deuxième délibération, qui comprend aussi l'introduction de la procession annuelle se terminant dans l'Asklépieion, qui apparaît au début du décret. Cependant, les institutions de Pergame décidèrent de garder dans la version finale du décret un résumé de la première délibération, celle concernant la fête d'accueil du roi : celle-ci constituait à ce moment-là un évènement passé, mais tout à fait digne d'être rappelé dans le document final, afin de préserver dans la mémoire civique le souvenir le plus complet de la générosité de Pergame envers son roi victorieux et bienfaiteur.

Cette distinction entre les deux parties du document a échappé aux commentateurs précédents, y compris Coarelli, qui interprète erronément la deuxième section du décret comme un texte prescriptif réglant le cérémoniel pour toute occasion de retour futur d'Attale ${ }^{98}$. Il en résulte que ce savant établit un parallèle, qui parait sans fondement, entre la fin de la cérémonie d'apantésis et la fin de la procession annuelle destinée à la commémorer, concluant qu'une célébration du banquet des magistrats 
dans le sanctuaire d'Asklépios hors-les-murs ne ferait aucun sens par rapport au mouvement d'entrée impliqué par l'accueil du roi. Au contraire, la topographie de la procession s'expliquerait, selon Coarelli, si on acceptait que l'apantésis se terminait dans l'Asklépieion situé dans la Theaterterrasse, près de l'agora où se tient le sacrifice au roi sur l'autel de Zeus Sôter (lignes 42-43) et près du palais royal où Attale se dirigerait ensuite ${ }^{99}$.

Néanmoins, il suffit de lire la séquence des événements conclusifs de l'apantésis (lignes 49-51), en gardant à l'esprit que ceux-ci décrivent un évènement passé, pour comprendre que cette cérémonie s'est terminée avec un banquet offert au roi par les stratèges dans le prytanée, probablement avec la viande sacrée d'une offrande de charistèria $^{100}$. En revanche, le banquet des magistrats à l'Asklépieion constitue la conclusion d'une cérémonie distincte et dont le mouvement, spéculaire à celui de la journée de l'apantésis, est motivé par le lien entre les honneurs cultuels pour Attale et le culte d'Asklépios. Ce lien, établi par la cité de Pergame pendant la deuxième phase de délibération, vise, comme d'autres initiatives prises à ce moment, à intégrer la mémoire des évènements dans le tissu de la vie religieuse de la communauté. On pourra donc conclure qu'à l'occasion des anniversaires de l'apantésis, la procession annuelle remettra en scène la descente de la population de Pergame vers le sanctuaire hors-lesmurs, où elle était sortie pour rencontrer le souverain. À cette occasion, Attale avait selon toute probabilité consacré une partie du butin de la victoire à Asklépios Sôter, l'épisode étant immortalisé par l'érection de la statue cultuelle représentant le roi cuirassé sur les armes des ennemis vaincus.

\section{L'époque impériale}

Pour revenir maintenant à la période impériale, il faut discuter le seul texte littéraire mentionnant de manière explicite un acte rituel adressé à Asklépios en relation avec la ville haute de Pergame. Il s'agit d'un passage de Fronton (Epist. 3.10.2) évoquant l'ascension du jeune Marc Aurèle à la citadelle pergaménienne pour supplier ce dieu en faveur de son maître : Igitur iam primum Pergami arcem ascendo et Aesculapio supplico, uti valetudinem magistri mei bene temperet vehementerque tueatur ${ }^{101}$. On peut remarquer que ce texte ne mentionne pas un lieu de culte d'Asklépios, mais se limite à faire allusion à une prière, qui ne doit pas nécessairement avoir lieu dans un temple de la divinité interpellée. Ainsi, la crédibilité de ce passage sur le plan topographique a été contestée, notamment par Müller, qui y a vu une indication vague de caractère rhétorique. On l'a vu, au $\mathrm{II}^{\mathrm{e}} \mathrm{s}$. ap. J.-C. Pergame était pour ainsi dire " pleine d'Asklépios $»^{102}$;cette divinité assurait à la ville son prestige international etla faveur impériale et le succès du culte montre un lien étroit avec les grandes initiatives évergétiques des élites civiques, qui assurèrent la monumentalisation du sanctuaire dans la vallée du Kaïkos $^{103}$. Si donc un étranger pouvait affirmer génériquement de se rendre à Pergame pour rencontrer le dieu, c'était le grandAsklépieion dans la vallée qui possédait les structures nécessaires à l'accomplissement rituel de cette expérience.

En partant de ces observations et en rappelant que la proximité entre les trois temples néocoriques dans les monnaies pergaméniennes de Caracalla peut être d'ordre symbolique plutôt que topographique, on est tenté de proposer que la statue du dieu trônant attestée dans ces monnaies - et déjà, comme on a vu, dans des types monétaires du règne d'Antonin le Pieux - ne constitue pas un attribut du temple de la 
Theaterterrasse, mais appartient à une nouvelle structure située à l'intérieur de l'Asklépieion dans la vallée. Déjà Burrell avait pensé identifier le temple hébergeant la statue d'Asklépios trônant dans les monnaies pergaméniennes des $\mathrm{II}^{\mathrm{e}}$ et III $\mathrm{e}^{\mathrm{e}}$ siècles ap. J.C.avec le temple de Zeus Asklépios, dédié par Rufinus au milieu du $\mathrm{II}^{\mathrm{e}} \mathrm{s}$. ap. J.-C., probablement entre la fin du règne d'Hadrien et celui d'Antonin le Pieux, dans la partie orientale du grand sanctuaire hors-les-murs. Elle abandonna pourtant cette piste en observant que même dans le type où le temple figure de trois quarts, l'édifice était toujours représenté comme un temple standard, alors que le temple de Rufinus comprenait une rotunda selon le modèle du Panthéon de Rome. Selon Burrell, la forme tout à fait insolite de ce temple aurait justifié une représentation mettant en valeur le caractère particulier du bâtiment, qui le rendait reconnaissable parmi tous les autres à Pergame ${ }^{104}$. Pourtant, en gardant à l'esprit les précautions méthodologiques contre une interprétation " photographique » des temples sur les monnaies, cette observation ne peut êt re considérée comme conclusive. On sait que le nombre de colonnes et le style de décoration pouvaient être représentés de manière simplifiée et idéaledans les monnaies, surtout si le but de l'iconographie était de mettre en valeur les détails de la statue cultuelle. Cette simplification fonctionnelle pourrait aussi avoir intéress é la représentation de l'architecture du temple de Rufinus dans les monnaies, si on pense que la nouvelle iconographie de la statue, avec la position du dieu trônant, exerçait une fonction encore plus importante que le plan du temple en relation à la définition du culte : elle traduisait visuellement la caractérisation du dieu comme Zeus Asklépios. Le lien entre cette nouvelle représentation du dieu et la consécration du nouveau temple par Rufinus est d'ailleurs confirmé chronologiquement par la réapparition de l'iconographie d'Asklépios trônant dans les monnaies pergaméniennes pendant lerègne d'Antonin le Pieux.

En conclusion, cette analyse détaillée de la documentation confirme la thèse selon laquelle les Pergaméniens ne construisirent jamais un deuxième Asklépieion à l'intérieur des murs, ni à la période hellénistique ni à la période impériale. Bien que la consécration d'un deuxième sanctuaire d'une divinité majeure soit attestée dans d'autres cités ${ }^{105}$, ceci ne parait pas être le cas de Pergame. La grandeur du programme architectural de l'Asklépieion hors-les-murs ainsi que la multiplication des inscriptions affichées dans ce sanctuaire au moment du plus grand prestige du culte d'Asklépios à Pergame, au $\mathrm{II}^{\mathrm{e}} \mathrm{s}$. ap. J.-C., montrent que cette structure, arrivée à un niveau de développement monumental sans précédents, catalysa à l'époque toute l'attention des élites civiques et des pèlerins étrangers, y compris les empereurs.

Cette conclusion implique aussi que, faute de nouveaux repères documentaires, l'identité du dieu vénéré dans le temple de la Theaterterrasse demeure toujours obscure. $\mathrm{Au}$ moins pour l'époque hellénistique, l'hypothèse de Dionysos Kathégémôn peut être retenue comme une solution plausible, en considération de l'utilisation de la terrasse qui abrite le temple comme plateforme d'accès au théâtre, bien qu'elle manque d'un appui documentaire décisif. Ce culte pourrait avoir survécu encore au début de la période impériale et jusqu'à l'incendie qui détruisit le temple en causant une interruption temporaire de son utilisation. Pourtant, comme l'a récemment proposé Posamentir, le temple semble avoir connu une re-fonctionnalisation après l'interruption de son utilisation ${ }^{106}$; l'occasion de cette re-fonctionnalisation fut offerte par la consécration du temple à Hadrien et Sabine en 124 ou 129. La nouvelle lecture de 
l'inscription sur l'épistyle du temple nous permet aussi d'éliminerraisonnablement cette structure du dossier concernant le troisième néocorat pour Caracalla, puisqu'on ne voit pas pourquoi ce changement de fonction ultérieur n'aurait pas été accompagné par une nouvelle dédicace inscrite.

Pour finir, le changement d'utilisation du temple dans la terrasse du théâtre apporte une confirmation ultérieure à un cadre interprétatif déjà bien caractérisé par la recherche récente sur Pergame: celui de la réorganisation - progressive mais substantielle - du paysage sacré de la cité qui accompagna la transition entre la période républicaine et l'époqueimpériale, en contraste avec la continuité plus marquée qui se manifeste entre les phases attalide et républicaine. Si cette continuité peut être entendue comme le signe d'une cité voulant s'accrocher à ses traditions régionales et à la splendeur de son passé attalide durant une époque troublée, le renouvellement de l'espace sacré de la Pergame impérialetémoigneen revanche de la capacité de la cité àinaugurer une nouvelle page de son identité et de s'engager activement, à travers les cultes d'Asklépios et des empereurs, dans son autopromotion dans le monde « global » et compétitif de l'Empire.

\section{BIBLIOGRAPHIE}

S. AGELISIS, « Kulte und Heiligtümer in Pergamon », in R. GRÜSSINGER, V. KÄSTNER, A. SCHOLL (éd.), Pergamon. Panorama der antiken Metropole, Berlin, 2011, p. 174-183.

R.E. ALLEN, The Attalid Kingdom : A Constitutional History, Oxford, 1983.

B. ANDREAE, « Der Asklepios des Phyromachos », in B. ANDREAE (éd.), Phyromachos-Probleme. Mit einem Anhang zur Datierung des Grossen Altares von Pergamon, Mainz, 1990, p. 46-100.

J. AUINGER, A. FENDT, « Die kolossale Kultbildgrupe aus dem Trajaneum », in R. GRÜSSINGER, V. KÄSTNER, A. SCHOLL (éd.), Pergamon. Panorama der antiken Metropole, Berlin, 2011, p. 167-171.

M. BACHMANN, « Topographie und Macht. Der Burgberg in Pergamon », in E.-L. SchWANDNER, K. RHEIDT (éd.), Macht der Architektur - Architektur der Macht. Bauforschungskolloquium Berlin 2002, Mainz, 2004, p. 121-131.

R. BIELFELDT, « Wo nur sind die Bürger von Pergamon? Eine Phänomenologie bürgerlicher Unscheinbarkeit im städtischen Raum des Königsresidenz », MDAI(I) 60 (2010), p. 117-201.

R. BOHN, Der Theaterterrasse, Berlin, 1896 (AvP IV).

Ch. BÖHRINGER, « Pergamon », in Neue deutsche Ausgrabungen in Mittelmeergebiet und im Vorderen Orient, Berlin, 1959, p. 121-171.

G.W. BOWERSOCK, « Greek Intellectuals and Imperial Cult in the Second Century A.D. », in

E. BICKERMAN (éd.), Le culte des souverains dans l'Empire romain, Vandœvres-Genève, 1983, p. 179-212

(Entretiens Hardt, 19).

B. BURRELL, Neokoroi : Greek Cities and Roman Emperors, Leiden/Boston, 2004. 
S.G. CANEVA, « Le retour d'Attale III à Pergame. Un réexamen du décret IvP I 246 », EA 51 (2018), p. 109-123.

-, «L'importance de la matérialité. Le rôle des petits objets inscrits dans la compréhension des cultes pour les souverains ", in S.G. CANEVA (éd.), The Materiality of Hellenistic Ruler Cults, Liège, 2020a, à paraître (Kernos, Suppl. 36).

-, « Les honneurs cultuels pour Attale III (IvP I 246) », in S.G. CANEVA (éd.), The Materiality of Hellenistic Ruler Cults, Liège, 2020b, à paraître (Kernos, Suppl. 36).

A. CHANIOTIS, Age of Conquests : The Greek World from Alexander to Hadrian (336 BC-AD 138), Cambridge, 2018.

A.S. CHANKOWSKI, « La procédure législative à Pergame au I ${ }^{\text {er }}$ siècle av J.-C. : à propos de la chronologie relative des décrets en l'honneur de Diodoros Pasparos », BCH 122.1 (1998), p. 159199.

-, « Les cultes des souverains hellénistiques après la disparition des dynasties : formes de survie et d'extinction d'une institution dans un contexte civique », in I. SAVALLI-LESTRADE, I. COGITORE, (éd.), Des Rois au Prince. Pratiques du pouvoir monarchique dans l'Orient hellénistique et romain (IVe av. J-C. - II ap. J.-C.), Grenoble, 2010, p. 271-290.

B. CHRUBASIK, D. KING (éd.), Hellenism and the Local Communities of the Eastern Mediterranean. 400 BCE$250 \mathrm{CE}$, Oxford/London, 2017.

F. COARELLI, Pergamo e il re. Forma e funzioni di una capitale ellenistica, Pise/Rome, 2016 (Studi Ellenistici, suppl. 3).

D. DAMASKOS, Untersuchungen zu hellenistischen Kultbildern, Stuttgart, 1999.

G. DE LUCA, Das Asklepieion. 4. Teil. Via Tecta und Hallenstraße. Die Funde, Berlin, 1984 (AvP XI.4).

-, « Asklepios in Pergamon », in B. ANDREAE (éd.), Phyromachos-Probleme. Mit einem Anhang zur Datierung des Grossen Altares von Pergamon, Mainz, 1990, p. 25-40.

—, « Zur Hygieia in Pergamon. Ein Beitrag », MDAI(I) 41 (1991), p. 325-345.

-, « Il culto di Asklepios in Asia Minore : il caso di Pergamo », in G. SFAMENI GASPARRo et al. (éd.), Il culto di Asclepio nell'area mediterranea. Atti del Convegno Internazionale, Agrigento 20-22 novembre 2005, Rome, 2009, p. 97-111.

G. DESPINIS, « Zu Akrolithstatuen griechischer und römischer Zeit », NachrAkGött 8 (2004), p. 245301.

Ch.P. DICKENSON, On the Agora : The Evolution of a Public Space in Hellenistic and Roman Greece (c. 323 BC - 267 AD), Leiden/Boston, 2017.

B. DIGNAS, « Rituals and the Construction of Identity in Attalid Pergamon », in B. DIGNAS, R.R.R. SMITH (éd.), Historical \& Religious Memory in the Ancient World, Oxford, 2012, p. 119-144.

W. DÖRPFELD, « Die Arbeiten zu Pergamon 1902-1903. Die Bauwerke », MDAI(A) 29 (1904), p. 113151.

-, « Die Arbeiten zu Pergamon 1906-1907. Die Bauwerke », MDAI(A) 33 (1908), p. 328-374.

-, « Die Arbeiten zu Pergamon 1908-1909. Die Bauwerke », MDAI(A) 35 (1910), p. 346-400.

B. EMME, Peristyl und Polis. Entwicklung und Funktionen öffentlicher griechischer Hofanlagen, Berlin, 2013. 
R. EVANS, A History of Pergamum : Beyond Hellenistic Kingship, London, 2012.

J.-L. FERRARY, Rome et le monde grec. Choix d'écrits, Paris, 2017.

F.R. FORSTER, Die Polis im Wandel. Ehrendekreten für eigene Bürger im Kontext der hellenistischen Polisgesellschaft, Göttingen, 2018 (Die hellenistische Polis als Lebensform, 9).

G. FRIJA, Les Prêtres des empereurs. Le culte impérial civique dans la province romaine d'Asie, Rennes, 2012.

Ph. GAUTHIER, Les cités grecques et leurs bienfaiteurs (IV $-I^{e r}$ siècle avant J.-C.) : contribution à l'histoire des institutions, Paris, 1985 (BCH, suppl. 12.).

H.-J. GEHRKE, Geschichte des Hellenismus, Munich, $2008^{4}$.

E. GRECO, compte rendu de COARELLI 2016, ASAtene XCIII S. III, 15 (2015), p. 325-328.

R. GRÜSSINGER, V. KÄSTNER, A. SCHOLL (éd.), Pergamon. Panorama der antiken Metropole, Berlin, 2011.

Ch. HABICHT, «Ist eine 'Honoratiorenregime' das Kenzeichen des Stadt im späteren

Hellenismus? », in M. WÖRRLE, P. ZANKER (éd.) Stadtbild und Bürgerbild im Hellenismus. Kolloquium, München, 24 bis 26 Juni 1993, Munich, 1995 (Vestigia, 47), p. 87-92.

P. HAMON, «Les prêtres du culte royal dans la capitale des Attalides : note sur le décret de Pergame en l'honneur du roi Attale III (OGIS 332)», Chiron 34 (2004), p. 169-185.

-, « Le Conseil et la participation des citoyens : les mutations de la basse époque hellénistique », in P. FRÖHLICH, Ch. MÜLLER(éd.), Citoyenneté et participation à la basse époque hellénistique. Actes de la table ronde des 22 et 23 mai 2004 (Paris), Genève/Paris, 2005, p. 121-144.

-, «Élites dirigeantes et processus d'aristocratisation à l'époque hellénistique », in H.-L. FERNOUX, Ch. STEIN(éd.), Aristocratie antique : modèles et exemplarité sociale. Actes de la Journée d'étude de Dijon, 25 novembre 2005, Dijon, 2007, p. 77-98.

A. HOFfmanN, « The Roman Remodeling of the Asklepieion », in H. KOESTER (éd.), Pergamon, Citadel of the Gods: Archaeological Record, Literary Description, and Religious Development, Harrisburg, 1998, p. 41-61.

A. HoffMAnN, G. DE LUCA, Das Asklepieion. 5. Teil. Die Platzhallen und die zugehörigen Annexbauten in römischer Zeit, Berlin, 2011 (AvP, XV.5).

A.-Fr. JACCOTTET, Choisir Dionysos. Les associations dionysiaques ou la face cachée du dionysisme, Zürich, 2003.

V. KÄSTNER, « Anfänge - Pergamon in vorattalidischer Zeit », in R. GRÜSSINGER, V. KÄSTNER, A. SCHOLL (éd.), Pergamon. Panorama der antiken Metropole, Berlin, 2011a, p. 28-33.

-, « Das Heiligtum von Athena », in R. GRÜSSINGER, V. KäSTNER, A. SCHOLL (éd.), Pergamon. Panorama der antiken Metropole, Berlin, 2011b, p. 184-193.

N. KAYE, "The Skeleton of the State": The Fiscal Politics of Pergamon, 188-133 B.C.E., PhD diss. University of California, Berkeley, 2012.

M. KLINKOTT, Die Stadtmauern. Teil 1. Die Byzantinischen Befestigungsanlagen von Pergamon, mit ihrer Wehr- und Baugeschichte, Berlin, 2001 (AvP XVI.1).

M. KOHL, « Das Nikephorion von Pergamon », Revue Archéologique 34 (2002), p. 227-253. 
-, « La Pergame d'Apollon depuis les temps de l'Iliade homérique à l'époque hellénistique », in M. конL(éd.),Pergame. Histoire et archéologie d'un centre urbain depuis ses origines jusqu'à la fin de l'antiquité, Lille, 2008, p. 147-169.

P. KRANZ, Pergameus Deus. Archaeologische und numismatische Studien zu den Darstellungen des Asklepios in Pergamon wahrend Hellenismus und Kaiserzeit; mit einem Exkurs zur Überlieferung statuarischer Bildwerke in der Antike, Möhnesee, 2004.

-, Hygieia - Die Frau an Asklepios' Seite. Untersuchungen zu Darstellung und Funktion in klassischer und hellenistischer Zeit unter Einbeziehung der Gestalt des Asklepios, Mo.'.hnesee, 2010.

Br. LE GUEN, Les associations de Technites dionysiaques à l'époque hellénistique, Nancy, 2001.

M. MAISCHBERGER, « Der Dionysos-Tempel auf der Theaterterrasse im Lichte neuer Forschung », in

R. GRÜSSINGER, V. KÄSTNER, A. SCHOLL (éd.), Pergamon. Panorama der antiken Metropole, Berlin, 2011, p. 243-247.

-, « Der Dionysos-Tempel auf der Theaterterrasse », in A. SCHoll, A. schWARZMAIER (éd.), Pergamon. Meisterwerke der antiken Metropolen und 360'-Panorama von Yadegar Asisi. Begleitbuch zur Ausstellung, Berlin, 2018, p. 120-125.

Ch. MANN, « Gleichheiten und Ungleichheiten in der hellenistischen Polis : Überlegungen zum Stadt der Forschung ", in Ch. MANN, P. scholz (éd.) "Demokratie” im Hellenismus. Von der Herrschaft des Volkes zur Herrschaft der Honoratioren?, Heidelberg, 2012 (Die hellenistische Polis als Lebensform, 2), p. 11-27.

M.-Ch. MARCELLESI, Pergame de la fin $d u v^{e}$ au début du I $I^{e r}$ siècle avant J.-C. : pratiques monétaires et histoire, Pise/Rome, 2012 (Studi Ellenistici, 26).

A. MATTHAEI, M. ZIMMERMANN (éd.), Stadtbilder im Hellenismus, Heidelberg, 2009 (Die hellenistische Polis als Lebensform, 1).

-, Urbane Strukturen und bürgerliche Identität im Hellenismus, Heidelberg, 2015 (Die hellenistische Polis als Lebensform, 5).

M. MELfI, « The Archaeology of the Asclepieum of Pergamum », in D.A. RusSELl et al. (éd.), In Praise of Asclepius : Aelius Aristides, Selected Prose Hymns, Tübingen, 2016, p. 89-113.

C. MICHELS, « Dionysos Kathegemon und der attalidische Herrscherkult. Überlegungen zur Herrschaftsrepräsentation der Könige von Pergamon », in L.-M. GUNTHER, S. PLISCHKE (éd.), Studien zum vorhellenistischen und hellenistischen Herrscherkult, Berlin, 2011, p. 114-140.

H. MÜLLER, « Phyromachos im pergamenischen Nikephorion? », Chiron 22 (1992), p. 195-226.

-, « Hadrian an die Pergamener. Eine Fallstudie », in R. HAENSCH (éd.), Selbdarstellung und Kommunikation. Die Veröffentlichung staatlicher Urkunden auf Stein und Bronze in der römischen Welt. Internationales Kolloquium an der Kommission für Alte Geschichte und Epigraphik in München, 1. bis 3. Juli 2006, Munich, 2009 (Vestigia, 61), p. 367-406.

-, « Römischer Kaiserkult », in R. GRÜSSINGER, V. KÄSTNER, A. SCHOLL (éd.), Pergamon. Panorama der antiken Metropole, Berlin, 2011, p. 153-157.

I. NIELSEN, « Die Räumlichkeiten für dionysische Vereine und ihre kulturellen, geschichtlichen und religiösen Kontexte ", in D. GRAEN, M. RIND, H. WABERSICH (éd.), Otium cum dignitate. Festschrift für Angelika Geyer zum 65. Geburtstag, Oxford, 2014 (BAR Int. Series, 2605), p. 49-60.

E. OHLEMUTZ, Die Kulte und Heiligtümer der Götter in Pergamon, Darmstadt, 1940. 
O. PALAGIA, «The Cult Statues of the Ptolemies and the Attalids », in S.G. CANEVA (éd.), The Materiality of Hellenistic Ruler Cults, Liège, 2020, à paraître (Kernos, Suppl. 36).

A. PALAMIDIS, L'abandon de sanctuaires et le transfert de cultes en Grèce antique, thèse de doctorat inédite, Université de Liège, 2017.

F. PIRSON, « Die Siedlungsgeschichte Pergamons - Überblick und kritische Revision », MDAI(I) 67 (2017), p. 43-130.

R. POSAMENTIR, « Der sogennante Caracalla-Tempel von Pergamon - ein Bau zu Ehren Hadrians und seiner Gattin Sabina », MDAI(I) 67 (2017), p. 187-236.

R. POSAMENTIR, H. WIENHOLZ, « Gebäude mit litterae aureae in den kleinasiatischen Provinzen, die Basilika von Berytus und der Jupitertempel von Baalbek », MDAI(I) 62 (2012), p. 161-198.

S.R.F. PRICE, Rituals and Power: The Roman Imperial Cult in Asia Minor, Cambridge, 1984.

F. QUEYREL, Les portraits des Attalides: fonction et représentation, Athènes, 2003.

-, L'autel de Pergame. Images et pouvoir en Grèce d'Asie, Paris, 2005.

W. RADT, « Zwei augusteische Dionysos-Altärchen aus Pergamon », in N. BAșGELEN, L. MIHIN (éd.), Festschrift für Jale Inan, Istanbul, 1989, p. 199-209.

-, Pergamon. Geschichte und Bauten einer antiken Metropole, Darmstadt, 1999.

W. RAECK, “Zur hellenistischen Bebauung der Akropolis von Pergamon », MDAI(I) 38 (1988), p. 201 236.

-, « Untersuchungen zur Vorgängerbebauung des Trajaneums von Pergamon I. Überblick, Obere Hangstufe », MDAI(I) 49 (1999), p. 333-356.

-, « Untersuchungen zur Vorgängerbebauung des Trajaneums von Pergamon II. Der Bereich westlich des Tempels, untere Hangstufe », MDAI(I) 50 (2000), p. 307-364.

G.H. RENBERG, Where Dreams May Come : Incubation Sanctuaries in the Greco-Roman World, Leiden/ Boston, 2016 (RGRW, 184).

-, «Prosopographical Problems Associated with the Establishment of Asklepios's Cult at Pergamon », ZPE 201 (2017), p. 155-159

K. RHEIDT, « Die Obere Agora. Zur Entwicklung des hellenistischen Stadtzentrums von Pergamon », MDAI(I) 42 (1992), p. 235-285.

-, « Polis und Stadtbild im 4. und 3. Jh. v.Chr. », in A. Matthaei, M. Zimmermann (éd.), Urbane Strukturen und bürgerliche Identität im Hellenismus, Heidelberg, 2015 (Die hellenistische Polis als Lebensform, 5), p. 300-329.

J.W. RIETHMÜLLER, Asklepios. Heiligtümer und Kulte, Heidelberg, 2005.

-, « Das Asklepieion von Pergamon », in R. GRÜSSInGER, V. KÄSTNER, A. SCHOLL (éd.), Pergamon. Panorama der antike Metropole, Berlin, 2011, p. 229-234.

K.J. RIGSBY, Asylia : Territorial Inviolability in the Hellenistic World, Berkeley et al., 1996.

L. ROBERT, Études anatoliennes, Paris, 1937.

-, « Un décret de Pergame », BCH 108 (1984), p. 472-489.

-, « Le décret de Pergame pour Attale III », BCH 109 (1985), p. 468-481.

D.A. RusSEll et al. (éd.), In Praise of Asclepius : Aelius Aristides, Selected Prose Hymns, Tübingen, 2016. 
B. SCHMIDT-DOUNAS, « Statuen hellenistischer Könige als Synnaoi Theoi », Egnatia 4 (1993-1994),

p. $71-132$.

E.-L. SCHWANDNER, « Beobachtungen zur hellenistischen Tempelarchitektur von Pergamon », in

W. HOEPFNER, E.-L. SCHWANDNER (éd.), Hermogenes und die hochhellenistische Architektur. Internationales Kolloquium in Berlin 1988, Mainz, 1990a, p. 85-102.

-, « Ein monumentaler dorischer Marmortempel in Pergamon », in B. ANDREAE (éd.), PhyromachosProbleme. Mit einem Anhang zur Datierung des Grossen Altares von Pergamon, Mainz, 1990b, p. 41-43.

E.-L. SCHWANDNER, K. RHEIDT (éd.), Macht der Architektur - Architektur der Macht, Mainz, 2004.

H. SCHWARZER, "Untersuchungen zum hellenistischen Herrscherkult in Pergamon ", MDAI(I) 49 (1999), p. 249-300.

-, « Der so-gennante Bau H - Zum mutmaßlichen Prytaneion von Pergamon », MDAI(I) 54 (2004), p. $173-183$.

-, « Die Bukoloi in Pergamon. Ein dionysischer Kultverein im Spiegel der archäologischen und epigraphischen Zeugnisse », in I. NIELSEN (éd.), Zwischen Kult und Gesellschaft : Kosmopolitische Zentren des antiken Mittelmeerraumes als Aktionsraum von Kultvereinen und Religionsgemeinschaften, Augsburg, 2006 (Hephaistos, 24), p. 153-167.

-, Das Gebäude mit dem Podiensaal in der Stadtgrabung von Pergamon. Studien zu sakralen Banketträumen mit Liegepodien in der Antike, Berlin 2008 (AvP, XV.4).

-, « Ein neu entdecktes marmornes Kolossalporträt eines Attaliden (Eumenes'II. ?) aus Pergamon », in H. SCHWARZER, H.-H. NIESWANDT (éd.), Man kann es sich nicht prächtig genug vorstellen! Festschrift für Dieter Salzmann zum 65. Geburtstag, Marsberg-Padberg, 2016, I, p. 351-358.

K.G. SIEGLER, « Ein hellenistischer Tempel des Zeus aus dem Burgberg von Pergamon? », in Koldewey-Gesellschaft. Bericht über die 32. Tagung für Ausgrabungswissenschaft und Bauforschung : vom 19.-23. Mai 1982 in Innsbruck, Bonn, 1984, p. 29-31.

B. SIELHORST, Hellenistische Agorai. Gestaltung, Rezeption und Semantik eines urbanen Raumes, Berlin, 2015.

J. STEINHAUER, Religious Associations in the Post-Classical Polis, Stuttgart, 2014.

D. Steuernagel, « Die Tempel aus der Zeit Attalidenherrschaft in Pergamon », in A. MATthaeI,

M. ZIMMERMANN (éd.), Urbane Strukturen und bürgerliche Identität im Hellenismus, Heidelberg, 2015, p. 360-385 (Die hellenistische Polis als Lebensform, 5).

A.F. STEWART, Attika. Studies in Athenian Scupture of the Hellenistic Age, London, 1979.

V.M. STROCKA, Das Markttor von Milet, Berlin, 1981 (Berlin WPr., 128).

-, « Wechselwirkungen der städtrömischen und kleinasiatischen Architektur unter Trajan und Hadrian », MDAI(I) 38, p. 291-307.

-, « Bauphasen der kaiserzeitlichen Asklepieions in Pergamon », MDAI(I) 62 (2012), p. 199-287.

P. THONEmAnN (éd.), Attalid Asia Minor: Money, International Relations, and the State, Oxford, 2013.

G. TOZZI, Assemblee politiche e spazio teatrale ad Atene, Padova, 2016.

O. VENTROUX, Les élites d'une ancienne capitale royale à l'époque romaine, Rennes, 2017.

B. VIRGILIO, « Eumene I e i mercenari di Filetereia e di Attaleia », SCO 32 (1982), p. 97-140.

-, Lancia, diadema e porpora. Il re e la regalità ellenistica, Pise/Rome, $2003^{2}$ (Studi Ellenistici, 14). 
R. VON DEN HOFF, « Das Gymnasion von Pergamon : herrscherlicher und bürgerlicher Raum in der hellenistischen Polis », in A. MATTHAEI, M. ZIMMERMANN (éd.), Urbane Strukturen und bürgerliche Identität im Hellenismus, Heidelberg, 2015, p. 123-145.

-, compte rendu de COARELLI 2016, Sehepunkte 17 (2017), $\mathrm{n}^{\circ} 5$.

-, « Bildnisse der Attaliden », in A. scholl, A. schwARZMAier (éd.), Pergamon. Meisterwerke der antiken Metropolen und $360^{\circ}$-Panorama von Yadegar Asisi. Begleitbuch zur Ausstellung, Berlin, 2018, p. 49-56.

H. VON FRITZE, « Asklepiosstatuen in Pergamon », Nomisma 2 (1908), p. 19-35.

-, Die Münzen von Pergamon, Berlin, 1910.

P. von JACOBSTHAL, « Die Arbeiten zu Pergamon 1906-1907 », MDAI(A) 33 (1908), p. 375-441.

H. vON PROTT, « Dionysos Kathegemon als Stammvater der Attaliden », MDAI(A) 27 (1902), p. 160188.

Ch. Williamson, « Power of Place: Ruler, Landscape, and Ritual Space at the Sanctuaries of Labraunda and Mamurt Kale in Asia Minor ", in C. MOSER, C. FELDMAN (éd.), Locating the Sacred: Theoretical Approaches to the Emplacement of Religion, Oxford/Oakville, 2014, p. 87-110.

F. WINTER, Die Skulpturen mit Ausnahme der Altarreliefs, Berlin, 1908 (AvP, VII.1).

M. WÖRRLE, « Pergamon um 300 v. Chr. », Chiron 30 (2000), p. 543-576.

-, « Zu Rang und Bedeutung von Gymnasion und Gymnasiarchie im hellenistischen Pergamon », Chiron 37 (2007), p. 501-517.

O. ZIEGENHAUS, Das Asklepieion. 3. Teil. Die Kultbauten aus römischer Zeit an der Ostseite des Heiligen Bezirks, Berlin, 1981 (AvP, XI.3).

O. ZIEGENHAUS, G. DE LUCA, Das Asklepieion. Der südliche Temenosbezirk in hellenistischer und frührömischer Zeit, Berlin, 1968 (AvP, XI.1).

-, Das Asklepieion. 2. Teil. Der nördliche Temenosbezirk und angrenzende Anlagen in hellenistischer un frührömischer Zeit, Berlin, 1975 (AvP, XI.2).

\section{NOTES}

1. Pour un encadrement thématique concernant en particulier la haute période hellénistique, cf. GEHRKE $\left(2008^{4}\right)$. Pour les traits caractérisant la basse période hellénistique, cf. GAUTHIER (1985) ; HABICHT (1995); HAMON (2005) et (2007); MANN (2012) (sur la place des élites dans les poleis); FERRARY (2017) (sur le rôle de Rome) ; FORSTER (2018).

2. Voir, tout récemment, CHRUBASIK - KING (2017) et CHANIOTIS (2018) (Ive s. av. J.-C. - II ${ }^{\mathrm{e}}$ ap. J.-C.). En ce qui concerne l'histoire religieuse, on peut aussi rappeler le projet Chronos, Chronologies des évolutions religieuses : formes religieuses, pratiques et agents cultuels (II ${ }^{e} . a v .-I^{e r}$ s. de notre ère), lancé en 2014 par le groupe de recherche parisien ANHIMA (http://www.anhima.fr/spip.php? article1162\&lang=fr).

3. Pour l'histoire de Pergame depuis les Attalides jusqu'à l'intégration dans la province d'Asie, ALLEN (1983) (histoire institutionnelle); KAYE (2012) et THONEMANN (2013) (rapports entre Pergame et l'Asie Mineure); MARCELLESI (2012) (histoire monétaire et financière). Pour une histoire de la cité de la fin de la dynastie (post 133 av. J.-C.) au Bas Empire, voir Evans (2012) pour les évènements politiques et militaires; ventroux (2017) pour l'histoire sociale des élites. Pour 
l'évolution des espaces publics, comme l'agora, le gymnase et les sanctuaires, sous la dynastie attalide, voir récemment SCHWANDNER - RHEIDT (2004) ; BIELFELDT (2010) ; MATTHAEI - ZIMMERMANN (2009) et (2015). En ce qui concerne le paysage sacré de Pergame entre la période hellénistique et impériale, voir les synthèses offertes par GRÜSSINGER - KÄSTNER - SCHOLL (2011), qui intègrent et mettent à jour RADT (1999), avec des conclusions très différentes des précédentes; COARELLI (2016) et PIRSON (2017) offrent maintenant une mise à jour fondamentale concernant l'évolution urbanistique de Pergame à la lumière des fouilles récentes dans la cité basse. KOHL (2008) et DIGNAS (2012) discutent les quelques traces des cultes de Pergame aux périodes archaïque et classique. À ce propos un cas particulièrement intéressant concerne le temple d'Athéna sur l'acropole, construit pendant le dernier tiers du $\mathrm{IV}^{\mathrm{e}}$ siècle et ensuite intégré dans le projet monumental d'Attale I $^{\text {er }}$ et Eumène II : RADT (1999), p. 159-167 ; KÄSTNER (2011a) et (2011b) ; COARELLI (2016), p. 61-77.

4. Du point de vue cultuel, les innovations les plus importantes de cette période de transition concernent la diffusion des petits sanctuaires rupestres mis au jour par les fouilles récentes: cf. PIRSON (2017), p. 46, 83, 92, 95.

5. RADT (1999), p. 200-208; AGELIDIS (2011), p. 182-183.

6. Sur le culte impérial comme opportunité d'autopromotion tant au niveau personnel que civique, cf. BOWERSOCK (1973); PRICE (1984); BURRELL (2004); MÜLLER (2011); FRIJA (2012). Sur le pouvoir d'attraction de l'Asklépieion dans le monde « global » de l'Empire, voir les contributions dédiées par RUSSELl et al. (2016) à Pergame dans l'œuvre d'Aelius Aristide.

7. Voir le cas révélateur d'Hiérôn, fils d'Asklépiadès, honoré pour avoir renouvelé les Sôtéria et les Hérakleia après une suspension de la fête causée par une guerre, probablement à identifier avec la guerre d'Andronique (MDAI(A) 33 [1908] p. 406, $\mathrm{n}^{\circ} 35$; IGR IV 300). La fête semble avoir disparu définitivement après 88 av. J.-C., à la conclusion de la guerre de Mithridate : cf. RIGSBY (1990), p. 380. Pour d'autres cas de bienfaiteurs privés pergaméniens honorés pour leurs interventions en faveur des cultes et des institutions de la cité, cf. CHANKowsKi (1998) (Diodoros Pasparos) ; WÖRRLE (2000) et (2007) ; FORSTER (2018), p. 209-224, 229-245. Sur le plan archéologique, le $\mathrm{I}^{\mathrm{er}}$ siècle av. J.-C. est caractérisé par une phase de stagnation et de déclin de l'Asklépieion: cf. MELFI (2016), p. 100. On notera pourtant que la nouvelle datation de l'expansion de la ville basse $\mathrm{au} \mathrm{I}^{\mathrm{er}} \mathrm{s}$. av. J.-C. révèle un dynamisme jusqu'ici méconnu, bien que ce phénomène s'encadre dans une phase historique négative pour la région, lorsque les tensions et les violences de la période post-attalide causèrent le transfert en ville d'une partie de la population de la vallée du Kaïkos en quête de sécurité : cf. PIRSON (2017), p. 92-95.

8. Cf. IGR IV 293b, lignes 7-9 et IGR IV 294, lignes 19-23 (statues des Attalides) ; IGR IV 294, lignes 47-48 (sacrifices aux souverains). Sur Diodoros et les honneurs pour les Attalides dans la Pergame républicaine, cf. CHANKOWSKI (1998) et (2010).

9. L'hypothèse d'un remplacement assez rapide des vieux destinataires du culte par les principes romains est confirmée, au niveau de l'initiative privée, par la documentation concernant le mobilier cultuel de la maison appelée par les archéologues allemands "das Gebäude mit dem Podiensaal ». Cf. schwARzer (2006) et (2008). Dès le début de l'époque impériale, cette maison hébergea l'association cultuelle des Boukoloi de Dionysos, comme le montre un autel dédié à Auguste par l'archiboukolos Hérodès: SEG XL 1136; JACCOTTET (2003), II p. 173-175, n ${ }^{\circ} 93$; SCHWARZER (2008), p. 236-237, cat. S1. Aux phases attalide tardive et républicaine de l'utilisation de la maison (phases 1-2: milieu du II ${ }^{\mathrm{e}}$ s. av. J.-C. - début du $\mathrm{I}^{\mathrm{er}} \mathrm{s}$ ap. J.-C.) appartiennent en revanche deux petits autels dédiés au culte d'Attale $\mathrm{I}^{\mathrm{er}}$ :RADT (1989), p. 204-205, nº 1 ;SEG XL 1134a ;SCHWARZER (2008), p. 237, cat. S21. Leur emplacement dans une maison construite entre la fin de la dynastie et le début de la période républicaine rend plausible l'hypothèse d'un culte posthume, ce qui est confirmé sur base paléographique par RADT $n^{\circ} 1$; cf. CANEVA (2020a). Le culte royal 
semble donc avoir survécu à la dynastie, mais non pas au changement de pouvoir causé par l'instauration du Principat.

10. COARELli (2016), p. 22, 55-59, 97, 104, suggérant que le temple d'Auguste et Rome, jamais localisé par les archéologues modernes (cf. BURRELL [2004], p. 17-22), soit en effet à identifier avec le temple d'Athéna Niképhoros. À ce propos, on peut noter que la représentation du temple d'Auguste comme un temple corinthien sur les monnaies pergaméniennes ne constitue pas en soi un argument décisif contre son identification avec le temple d'Athéna, qui était d'ordre dorique. Ce détail s'explique plutôt par rapport à une tendance à la stylisation, commune dans l'iconographie numismatique, qui met souvent en scène l'image d'un temple «standard » de l'époque, s'appuyant sur d'autres éléments (par exemple, la statue cultuelle ou des attributs divins) pour rendre l'édifice reconnaissable. Voir à cet égard la discussion de quelques cas concrets plus avant (n. 62-64).

11. Sur le culte néocorique de Trajan et Zeus Philios, introduit probablement en 113 ap. J.-C., voir BURRELL (2004), p. 22-30 ; MÜLLER (2011), p. 154-157. SIEGLER (1984) a proposé de reconstruire les traces d'un temple hellénistique sous le Traianeum; voir pourtant les critiques de RAECK (1988), p. 207 et 218, n. 54 ; RAECK (1999), p. 341, n. 17 ; RAECK (2000), p. 333, n. 40. L'hypothèse de Siegler est acceptée et développée par COARELLI (2016), p. 41-49, qui propose de voir dans ce temple un lieu de culte joint de Zeus Philios et Eumène II (cf. l'Euméneion mentionné dans IvP I 240).

12. Le texte a été republié avec des lectures différentes dans OGIS 332 et Virgilio (2003²), p. 246251, no 14 ; HAMON (2004) et CANEVA (2018) apportent des corrections ponctuelles. Comme j'ai montré dans CANEVA (2018), le texte préservé combine deux phases distinctes de délibération par les institutions de Pergame, avant et après le retour d'Attale. Pour une discussion plus détaillée des honneurs cultuels accordés à Attale III, cf. CANEVA (2020b).

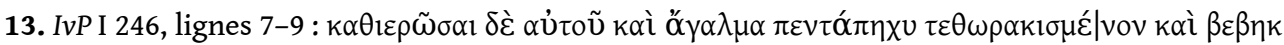

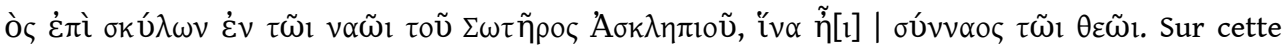
statue, voir PALAGIA (2020), aves les références antérieures.

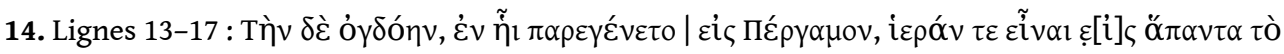

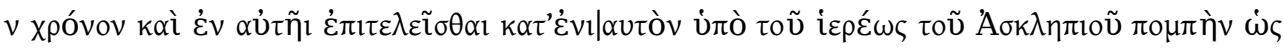

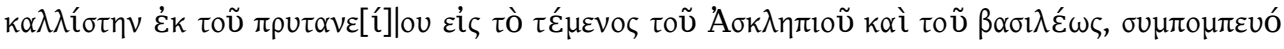



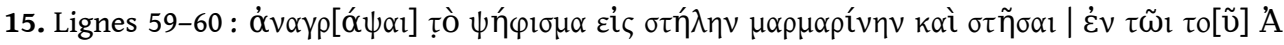

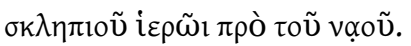

16. À ce propos, cf. ROBERT (1984) et (1985).

17. La date précise et les acteurs de l'installation du culte d'Asklépios dans la plaine en face de Pergame demeurent des questions débattues. Il en va de même pour l'identité du (ou des) destinataire(s) des activités cultuelles qui, selon toute vraisemblance, étaient pratiquées en ce lieu déjà avant l'arrivée d'Asklépios. À ce propos, voir les synthèses de RIETHMÜLLER (2005), I, p. 336-340 ; STEUERNAGEL (2015), p. 368-369 ; MELFI (2016), p. 90-91; RENBERG (2016), I p. 181, n. 153, et (2017).

18. Sur l'histoire du sanctuaire, en plus des publications des fouilles allemandes (AvP XI.1-5), voir, entre autres, HOFFMANN (1998); DE LUCA (2009) ; RIETHMÜLLER (2005), I, p. 334-359 et (2011) ; STROCKA (2012) ; RENBERG (2016), I, p. 138-146, 192-202 ; MELFI (2016).

19. Soit déjà sous Philétaire, soit sous son successeur Eumène $\mathrm{i}^{\mathrm{er}}$. Ce repère chronologique se fonde sur une comparaison stylistique entre la décoration d'un fragment en marbre appartenant à un temple dorique, remplacé ensuite par le temple ionique qui restera en place jusqu'à l'époque impériale, et le style du sanctuaire de Déméter, consacré par Philétaire et Eumène $\mathrm{I}^{\mathrm{er}}$ sur le versant sud-ouest de la colline de Pergame : cf. zIEGENHAUS - DE LUCA (1975), p. 79 et Taf. 34d; RIETHMÜLLER (2011), p. 345 ; STEUERNAGel (2015), p. 368. Pour une synthèse des Bauphasen 4-8, datées de la période entre Philétaire et Attale I ${ }^{\mathrm{er}}$ (phases 4-8), voir RIETHMÜLLER (2005), I, p. 340-352. 
20. Pour la datation traditionnelle de cette phase sous Hadrien, voir HOFFMANN (1998) et HOFFMANN - DE LUCA (2011). Selon STRocka (2012), en revanche, la grande phase d'expansion et de renouvellement du sanctuaire aurait déjà commencé sous la dynastie flavienne pour se conclure sous Hadrien. Voir aussi COARELLI (2016), p. 234-244 ; MELFI (2016), p. 101-103.

21. Sur cet édifice, situé directement au sud des temples dès la phase de construction 4 , voir ZIEGENHAUS - DELUCA (1975), p.17-19; récemment, RENBERG (2016), I, p.138-146, avec une discussion de la bibliographie antérieure.

22. Le premier bâtiment de ce type est le Nordostbau, situé à l'est de la Felsbarre : cf. ziEGENHAUS DE LUCA (1975), p. 24-26 ; RIETHMÜLLER (2005), I, p. 341, 359 et (2011), p. 231 ; MELFI (2016), p. 94-95 (Bauphase 5 , deuxième tiers du $\mathrm{III}^{\mathrm{e}}$ siècle). Une autre structure vraisemblablement destinée aux banquets est reconnue dans la Osthöfe, un édifice situé à l'est de la Festplatz du sanctuaire préromain, directement au sud du Nordostbau. L'édifice, appartenant à la phase de construction 10, est daté du premier quart du II ${ }^{\mathrm{e}}$ siècle, sous Eumène II : cf. ZIEGENHAUS - DE LUCA (1975), p. 47-55 ; RIETHMÜLLER (2005), I, p. 358.

23. RIETHMÜLLER (2011), p. 340-341.

24. Pour le modèle hécatomnide, voir parmi d'autres WILLIAMSON (2014) ; STEUERNAGEL (2015).

25. Voir RIETHMÜLLER (2005), I, p. 357-359 pour le programme monumental du règne d'Eumène II, correspondant aux Bauphasen 9-10.

26. Pour nous limiter au temple d'Asklépios, l'hypothèse de ZIEGENHAUS - DE LUCA (1975) (cf. RADT [1999], p. 220), qui identifient le temple avec les restes de l'édifice sud, est rejetée par RIETHMÜLLER (2005), I, p. 347-351 (cf. RIETHMÜLLER [2011], p. 231-232), qui considère comme plus probable une identification des fondations situées au milieu de la Felsbarre avec celles du temple d'Asklépios, peut-être vénéré avec Hygieia (IVP III 158). Sur la présence d'Hygieia à Pergame, voir aussi DE LUCA (1991) ; KRANZ (2010), p. 93-101, 149-161.

27. VON FRITZE (1908) et (1910).

28. VON PROTT (1902). Récemment, cette identification est soutenue, entre autres, par MAISCHBERGER (2011) et (2018) ; STEUernagel (2015), p. 368 (« sehr wahrscheinlich»). Pourtant, POSAMEnTIR (2017), p. 214, 222-223 exprime quelques réserves à propos de l'existence d'un culte de Dionysos dans ce temple, au moins après le renouvellement de la période impériale. Son point de vue est repris par MAISCHBERGER (2018), p. 124-125.

29. Voir MÜLLER (1992), sur lequel on reviendra plus loin.

30. CORARelli (2016), p. 35-41 a proposé d'interpréter le temple $\mathrm{R}$ comme un lieu de culte de Dionysos. Ne pouvant discuter ici l'ensemble de la documentation en détail, je me limiterai à partager les réserves exprimées par VON DEN HOFF (2017).

31. DÖRPFELD (1908), p. 354-355.

32. ANDREAE (1990). Pour l'hypothèse du déplacement et de la réutilisation de fragments architecturaux provenant d'un autre temple dans le cadre de la réalisation du temple $\mathrm{R}$, voir SCHWANDNER (1990a) et (1990b). Datée habituellement de la deuxième moitié du III siècle, la carrière de Phyromachos se situerait, selon Andreae, dans les années 182-156 (entre la fondation du Niképhorion par Eumène II et sa destruction par Prousias II), ce qui ferait de cet artiste un modèle direct et crucial de tout le style «baroque " pergaménien des règnes d'Eumène II et Attale II.

33. L'identification du Niképhorion dans la vallée du Kaïkos demeure problématique. BÖHRINGER (1959), p. 141-152, suivi par RADT (1999), p. 242-243, localise de manière hypothétique ce sanctuaire sur la colline de Muzalla Mezarlı̆̆ı, au sud de l'amphithéâtre romain dans la vallée en face de Pergame. Selon ANDREAE (1990), p. 70-72, 87-89, le temple d'Asklépios hébergeant la statue de Phyromachos se situerait dans le Niképhorion et serait à distinguer du temple de l'Asklépieion sur la Felsbarre; contra, voir les observations de MÜLLER (1992), p. 202-206, qui réaffirme l'unicité 
du sanctuaire d'Asklépios. Peu convaincante, car en contradiction avec la topographie suggérée par les textes anciens, est la thèse de конL (2002), identifiant le Niképhorion avec le sanctuaire d'Athéna Niképhoros sur la citadelle de Pergame. Récemment, en s'appuyant sur la totalité des sources textuelles concernant les attaques de Philippe V et de Prousias (cf. en particulier Polyb. 16.1.5 et 32.15.1-4; Diod. 31 fr. 51 GOUKOWSKI), COARELLI (2016), p. 222-234 est revenu sur la question avec une nouvelle interprétation: le Niképhorion serait le nom attribué, dans les sources littéraires, à un complexe sacré comprenant à la fois l'Asklépieion et d'autres sanctuaires, en particulier un sanctuaire d'Aphrodite. L'hypothèse est fascinante, bien qu'elle ne résolve pas toutes les questions ouvertes : par exemple, Polybe (18.2.2) mentionne en séquence le Niképhorion et le sanctuaire d'Aphrodite, de manière paratactique, ce qui semble contredire l'idée que le deuxième faisait partie du premier. De plus, l'affirmation que le nom Asklépieion n'apparaît pas dans les sources avant la fin de la dynastie (COARELLI [2016], p. 234) est à nuancer à la lumière du décret IvP I 246 pour Attale III, qui mentionne les revenus de ce sanctuaire (ligne

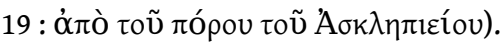

34. Polyb. 32.15.1-2 ; Diod. 31 fr. 51 GouKowsKI ; cf. App., Mithr. 3.7.

35. OHLEMUTZ (1940), p. 128-129.

36. MÜLLER (1992), en particulier p. 212-223 pour ce qui concerne la documentation du temple R. Dans son étude des sanctuaires d'Asklépios, RIETHMÜLLER (2005), I, p. 351-357 revient sur la thèse d'Andraeae pour la rejeter en ce qui concerne la datation tardive et l'iconographie (avec le dieu trônant, contra le reste des chercheurs qui pensent à une figure débout) de la statue cultuelle réalisée par l'artiste Phyromachos. De manière surprenante, RIETHMÜLLER (2005), I, p. 344, 354355, n. 205, et II p. 354, $\mathrm{n}^{\circ} 235$ cite l'article de Müller, mais contre toute évidence accepte l'identification du temple R avec un Asklépieion. Il faut également rectifier la référence (I, p. 354) au décret pergaménien OGIS 332 (IVP II 246) comme à un texte d'Élaïa, selon une hypothèse déjà rejetée de manière définitive par ROBERT (1984) et (1985).

37. En ce qui concerne le contenu, un texte très mutilé gravé sur un bloc positionné derrière

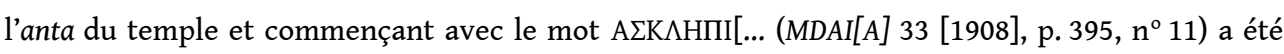
longtemps interprété comme le début d'une dédicace à Asklépios, alors que nous avons à faire avec le nom personnel d'un "prytane et prêtre (de Philétaire) », le magistrat éponyme de Pergame, dans la formule d'ouverture d'une liste d'éphèbes de la période post-attalide : cf. MÜLLER (1992), p. 219-222. Une autre liste d'éphèbes post-attalide, affichée sur un bloc du mur du temple R (MDAIII 33 [1908], p. 389, $\mathrm{n}^{\circ}$ 6, ligne 6), préserve le nom d'Asklépios Sôter comme gymnasiarque. Comme l'a montré roBert (1937), p. 70-71, n. 8, il s'agit d'une solution adoptée lorsqu'aucun citoyen n'acceptait d'assumer la charge de gymnasiarque (et les dépenses qui lui étaient attachées). Comme l'inscription précédente, cette liste ne témoigne pas d'un culte d'Asklépios pratiqué dans le temple $\mathrm{R}$, mais bien de l'importance de ce dieu pour les jeunes membres du gymnase dans la Pergame post-attalide, ainsi que de leur utilisation du temple $\mathrm{R}$ comme lieu d'affichage des listes éphébiques : cf. MÜLLER (1992), p. 218-219. Enfin, le règlement rituel IvP II 264 (LSAM 14) trouvé sur la terrasse entre le sanctuaire de Déméter et le temple R, au nord-ouest du gymnase, est daté du II ${ }^{\mathrm{e}}$ siècle ap. J.-C. et concerne les normes de pureté pour l'accès à l'incubation dans le grand Asklépieion dans la vallée du Kaïkos. Ni le temple R, ni aucun autre temple à l'intérieur des murs ne présente une structure adaptée à abriter un rituel d'incubation : à ce propos, voir déjà OHLEMUTZ (1940), p. 172 ; WÖRRLE dans IVP III, p. 180, n. 64 ; MÜLLER (1992), p. 217-218 ; RENBERG (2016), I p. 196-197.

38. Pour cette interprétation, voir VON JACOBSTHAL (1908), p. 421-422, selon lequel la statue serait tombée de la terrasse du temple R ; cf. OHLEMUTZ (1940), p. 128-129 ; DE LUCA (1990), p. 30-33. RADT (1999), p. 130-131 laisse la question de l'identification ouverte, tout en gardant l'appartenance de la statue au temple, qui n'est pourtant pas sûre : de fait, la statue pourrait aussi avoir fait partie de la décoration des thermes romains. D'autres identifications ont été avancées avec Zeus, un 
Attalide divinisé, ou Héraclès : cf. ROBERT (1937), p. 70-71, n. 8 ; MÜLLER (1992), p. 216 ; DAMASKOS (1999), p. 150-151.

39. Pour un catalogue et une analyse des différents lieux de découverte, voir OHLEMUTZ (1940), p. 129, 156-158 ; MÜLLER (1992), p. 216-217 ; RIETHMÜLLER (2005), I, p. 354-355, n. 205 et II, p. 363364. Un contexte domestique est assuré pour certains exemplaires trouvés en emplacement primaire (cf. W. RADT in AA 1985.3) et pourrait convenir à d'autres pièces pour lesquelles le contexte de dédicace originaire est moins sûr, voir inconnu : cf. KRANZ (2004), p. 26-30.

40. STEUERNAGEL (2015), p. 364-365, n. 27.

41. MÜLLER (1992), p. 225 ; STEUERNAGEL (2015), p. 364-365. La diffusion de l'ordre ionique se révèle être un trait distinctif du goût de cette époque et la transformation du programme décoratif d'un temple de l'ordre dorique en l'ordre ionique trouve un parallèle étroit dans l'Asklépieion horsles-murs.

42. Cf. Steuernagel (2015), p. 374, indiquant une phase de passage dans les expérimentations stylistiques (ordre dorique avec influences ioniques) du temple de Zeus Sôter dans l'agora (règne d'Attale $\mathrm{I}^{\mathrm{er}}$, dernier tiers $\mathrm{du}_{\mathrm{III}} \mathrm{e}^{\mathrm{e}}$ siècle). Pour l'origine méridionale de ces expérimentations, à situer dans la Carie des Hécatomnides et à Milet au milieu du IV siècle, cf. KÄSTNER (2011a), p. 3132.

43. Celle-ci fut à son tour divisée en deux terrasses, au plus tard sous Eumène II : celle du sanctuaire de Zeus, l'agora proprement dite, au sud, et celle du Grand Autel au nord. Sur l'évolution diachronique de l'agora supérieure, voir RHEIDT (1992) et (2015) ; BIELFELDT (2010), p. 168-182; PIRSON (2017), p. 63.

44. Pour le petit autel d'Asklépios et Hygieia, voir la discussion ci-dessous. Une deuxième inscription, appartenant à la phase impériale du temple, fut trouvée à l'intérieur de l'édifice. Il s'agit d'un texte presqu'illisible, inscrit sur l'épistyle du naiksos qui hébergeait la statue (ou plus probablement, les deux statues) de culte : cf. BOHN (1885), p. 55-56 ; POSAMENTIR (2017), p. 222-222. Les lettres préservées, $\Delta[$ c. 3 ?] $][$ [...]EI, ont été complétées de manière différente, et très hypothétique, en fonction de la thèse adoptée par chaque savant concernant l'identification du dieu vénéré dans le temple. Dans le cadre de sa reconstruction du culte de Dionysos chez les



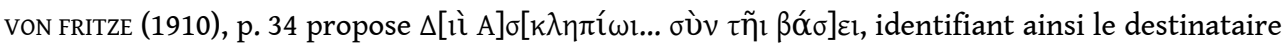
du culte avec Asklépios, et précisément Zeus Asklépios, une appellation connue de l'Asklépieion hors-les-murs pendant la phase impériale. Il est évident que d'autres intégrations pourraient également être possibles.

45. Sur la chronologie de l'érection du temple, voir BOHN (1896), p. 74 ; RADT (1999), p. 189 (probablement fin du III ${ }^{\mathrm{e}}$ siècle) ; BACHMANN (2004), p 126-127; MAISCHBERGER (2011) (fin du III ${ }^{\mathrm{e}}$ ou plus probablement première moitié du $\mathrm{II}^{\mathrm{e}}$, sous Eumène II) ; STEUERNAGEL (2015), p. 369-370.

46. STEUERNAGEL (2015), p. 369-370.

47. BOHN (1896), p. 66-67 ; cf. MAISCHBERGER (2011), p. 246 ; POSAMENTIR (2017), p. 190-194, 200, 218 -

219.

48. BURRELL (2004), p. 32 ; cf. COARELLI (2016), p. 28.

49. FRÄNKEL dans IvP II, p. 226.

50. BURRELL (2004), p. 30-35.

51. STROCKA (1981), p. 31, 54 n. 93 et (1988), p. 297-299 ; MAISCHBERGER (2011), p. 247 ; POSAMENTIR WIENHOLZ (2012), p. 162-163, n. 12 ; POSAMENTIR (2017), p. 193-194. En essayant de désamorcer ces conclusions, COARELLI (2016), p. 28, n.57, avance deux explications alternatives pour les similitudes entre les fragments du temple de la Theaterterrasse et les décorations du Traianeum : soit on aurait affaire ici à un cas d'imitation directe du Traianeum, soit on pourrait penser que les blocs en question, traditionnellement attribués au temple ionique de la terrasse du théâtre, soient en fait tombés de la terrasse du Traianeum. Ces deux hypothèses demeurent également 
spéculatives et les arguments stylistiques en faveur d'une reconstruction sous Hadrien sont réaffirmés par VON DEN HOFF (2017) dans le compte rendu du livre de Coarelli.

52. POSAMENTIR (2017); à la p. 213 sont discutés les restes très fragmentaires d'une deuxième ligne, d'une dimension plus petite, qui pourrait avoir contenu une référence à l'initiative évergétique de quelques membres de l'élite civique de Pergame.

53. MÜLLER (2009) = SEG LIX 1424; An. Ép. (2009) [2012], nº 1381 (texte et traduction française). Voir aussi MÜLLER (2011), p. 157. Sur les raisons de cette initiative, visant à augmenter le prestige de Pergame au détriment de Smyrne et d'Éphèse, les autres villes titulaires de deux temples néocoriques dans la province d'Asie, voir aussi POSAMENTIR (2017), p. 214-221.

54. Cf. POSAMENTIR (2017), p. 218, pour un possible rapprochement entre ce temple et l'Hadrianeum mentionné dans Ael. Arist. 47.29. Cette hypothèse d'identification n'est pourtant pas convaincante : l'édifice mentionné parAelius Aristide devait contenir un espace où le visiteur pouvait se baigner, ce qui ne convient pas au temple de la Theaterterrasse, mais bien au sanctuaire d'Asklépios (cf. IvP III, p. 10). Rappelons qu'à Pergame,une inscription trouvée dans l'Asklépieion définit Hadrien Néos Asklépios (IvP II 365, ligne 5 ; cf. venTROux [2017], p. 76-77). Sur l'Hadrianeum, voir aussi BURRELL (2004), p. 28 ; STROCKA (2012), p. 242 ;COARELLI (2016), p. 250.

55. Berlin, Antikensammlung SMB, AvP VII 281 (Trajan) et VII 282 (Hadrien), avec MÜLLER (2011), p. 157 ; AUINGER - FENDT (2011). On notera l'escamotage rhétorique d'Hadrien, qui, dans sa lettre, utilise la formule générique $i \delta \rho[\tilde{v} \sigma \alpha]-; \mu \varepsilon$ nol ("ériger [un portrait de] moi quelque part »; ligne 24), évitant toute implication concernant le statut de la statue, qu'elle soit honorifique ou cultuelle.

56. COARELLI (2016), p. 15-35, en particulier p. 26-27 pour la discussion du dossier numismatique (avec une riche bibliographie antérieure).

57. VON FRITZE (1908), p. 28-35, et (1910), p. 50-51.

58. À cet égard, on notera que les conclusions de MÜLLER (1992) contre l'existence d'un deuxième Asklépieion (suivies par HAMON [2004], p. 173-174) concernent seulement le temple R.

59. OHLEMUTZ (1940), p. 127, n. 8 pour le temple R.

60. PRICE (1984), p. 152 et 253, no 23 à propos du temple de la Theaterterrasse.

61. Selon une hypothèse déjà développée par STEWART (1979), l'iconographie du dieu assis renverrait directement au modèle de la statue cultuelle de l'Asklépieion d'Épidaure, d'où le culte du dieu guérisseur avait été importé à Pergame. Cette interprétation est acceptée par coAReLLI (2016), p. 26, mais plusieurs savants ont noté qu'elle doit être nuancée à la lumière de la documentation sculpturale et monétaire de la période hellénistique : cf. ci-dessous, n. 66-67.

62. Les détails de ce temple sur les monnaies posent plusieurs problèmes interprétatifs: le temple est en effet représenté comme hexastyle, mais aucun temple d'Asklépios à Pergame ne correspond à cette description : cf. BURRELL (2004), p. 33. Quelques exemples concrets permettent de conclure que le nombre de colonnes n'est pas censé évoquer un détail précis, mais représente un temple idéal ; c'est la statue du dieu, en revanche, qui permet d'identifier le temple. À cet égard, voir la monnaie Pergamon 164 (règne d'Antoninus Pius) de la Staatliche Münzsammlung de Munich, où le dieu debout figure dans un temple tétrastyle, correspondant à la réalité du temple de la Felsbarre : cf. vON FRIETZE (1910), p. 47 ; KRANZ (2004), p. 45-46, fig. 36. Sur la monnaie Berlin, Münzkabinett SMC, IKMK 18230151 (MÜLLER [2011], p. 154), du règne de Trajan, le temple d'Auguste et celui de Trajan, représentés respectivement sur le recto et sur le verso, présentent tous les deux quatre colonnes corinthiennes. Dans la mesure où le Traianeum est un temple hexastyle, le nombre de colonnes est sans doute un choix idéalisant visant à souligner un parallélisme entre les deux temples. BURRELL (2004), p. 19, parle à ce propos d'une simplification numismatique justifiée par l'objectif de mettre en valeur l'image de la statue cultuelle à l'intérieur du temple.

63. Le temple peut apparaître frontalement ou de trois quarts : cf. BURRELL (2004), p. 31 ; COARELLI (2016), p. 24, fig. 23. 
64. Encore une fois, il faudra s'abstenir de tirer des conclusions à partir des détails des temples tels qu'ils apparaissent sur les monnaies: cf. coAReLli (2016), p. 24, fig. 22, avec trois temples tétrastyles corinthiens, à confronter avec BURRELL (2004), p. 31, type 22 (fig. 56), avec trois temples corinthiens, dont celui d'Asklépios à quatre colonnes, mais entouré par les deux autres temples qui sont représentés comme hexastyles.

65. Voir à ce propos le cas d'Éphèse discuté parBURRELL (2004), p. 70, 76-77. Cette possibilité est aussi reconnue par COARELLI (2016), p. 55.

66. KRANZ (2004), p. 44, 101 ; cf. DE LUCA (1990), 26-28, 34 contra STEWART (1979) ; BURREL (2004), p. 32.

67. KRANZ (2004), p. 101.

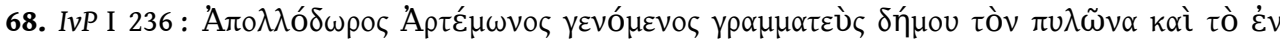


dynastie. La double référence au dieu et au peuple est expliquée par BIELFELDT (2010), p. 184, en relation à l'utilisation du théâtre pour les représentations scéniques et pour l'ekklésia. Pour cette deuxième fonction des théâtres hellénistiques, voir maintenant l'étude deтоzzi (2016).

69. Évidemment, ce raisonnement ne permet pas non plus d'exclure l'hypothèse qu'un temple dionysiaque pût avoir été consacré près du théâtre. En faveur de cette hypothèse, on notera que la terrasse du théâtre continua de jouer un rôle fondamental dans l'activité des Boukoloi de Dionysos Kathégémôn à la haute époque impériale ( $\mathrm{I}^{\mathrm{er}}$ - première moitié du $\mathrm{II}^{\mathrm{e}} \mathrm{s}$. ap. J.-C.). Comme le montre le dossier épigraphique - cf. en particulier JACCOTTET (2003), II $n^{\circ} 94$ et 99 ; schWARzER (2006) - cette association avait une fonction de premier rang non seulement dans les performances artistiques du théâtre, mais aussi dans les mystères du dieu. Il faut d'ailleurs rejeter l'argument de COARELLI (2016), p. 30, selon lequel le temple effectivement lié au théâtre ne serait pas celui de la terrasse, mais bien le sanctuaire d'Athéna. Cette observation contrintuitive est démentie par le fait que l'accès au théâtre se faisait sans doute par cette terrasse basse (comme le montre d'ailleurs le portal dédié par Apollodore), et non pas par l'extrémité escarpée de la terrasse d'Athéna. La proposition (COARELli [2016], p. 172-175) de voir dans la terrasse du théâtre la piste athlétique utilisée pour les concours des panégyreis, mentionnée dans les inscriptions, semble en revanche plus intéressante.

70. Plusieurs exemples sont offerts par les dédicaces privées publiées dans IvP II (période postattalide), section III B, Weihungen aussen denen an Kaiser.

71. COARELLI (2016), p. 31-33. L'identification avec le dernier Attalide est acceptée par SCHWARZER (2016), p. 355-356 ; PALAGIA (2020) ; contra QUEYREL (2003), p. 148-153, qui propose de manière peu convaincante Eumène II.

72. COARELLI (2016), p. 31. Le calcul se fonde sur une estimation du rapport de 1/10 entre le visage et le corps.

73. COARELLI (2016), p. 33.

74. L'indication de WINTER (1908), p. 150 (“im ionischen Tempel in der Mitte der Rückwand, zwei Meter über dem Fußboden"), révèle que le portrait fut retrouvé en contexte de remploi.

75. QUEYREL (2003), p. 149 pour le visage du temple ionique. Sur les acrolithes dans la sculpture sacrée grecque, voir l'étude générale de DESPINIS (2004) ; cf. PALAGIA (2020) pour les statues de culte des Ptolémées et des Attalides.

76. Cf. plus haut, n. 8 .

77. Voir QUEYREL (2003), en particulier p. 244-247, cat. F1-F2, pour deux autres fragments de statues que l'on pourrait attribuer à Attale III : ils furent dégagés respectivement dans un mur sur le versant sud de l'acropole (probablement près de son lieu d'origine) et dans le gymnase. Sur les portraits d'Attalides dans le gymnase, cf. maintenant VON DEN HOFF (2015), p. 127-130 et (2018), p. 54-55. Voir aussi la statue, probablement d'Attale II en Zeus, dans le sanctuaire d'Héra: SCHMIDT-DOUNAS (1994), p. 105-107 ; COARELL (2016), p. 50. On peut ajouter à ce dossier le fragment 
d'un nouveau visage d'Attalide, découvert hors contexte mais possiblement appartenant à une autre statue acrolithe ; selon l'éditeur, SCHWARZER (2016), il s'agirait d'Eumène II.

78. COARELli (2016), p. 33-34. Pour ce téménos, situé « près du théâtre » (de Pergame, ou plutôt de Téos ?), voir OGIS 326 (CIG 3069; LE GUEN [2001], nº 52). Le dossier concernant les Attalistai est recueilli et commenté par LE GUEN (2001), p. 253-265, nº 49-52 ; voir aussi SCHWARZER (1999), p. $266-$ 268 ; MiCHELS (2011), p. 130-131; POSAMENTIR (2017), p. 223-224.

79. Ainsi aussi SCHWARZER (1999), p. 270-272.

80. Cette donation testamentaire concerne aussi une maison privée, de l'argent, des esclaves et des objets nécessaires pour les rituels accomplis par l'association : cf. OGIS 326, lignes 20-32.

81. Voir l'étude de Steinhauer (2014), en particulier p.110-140. Pour les associations dionysiaques, cf. JACCOTTET (2003) ; SCHWARZER (2006) et (2008) ; NIELSEN (2014).

82. Sur ce monument, voir OHLEMUTZ (1940), p. 102-104 ; RADT (1999), p. 193-196.

83. Le texte est aussi publié dans OGIS 266. Pour une analyse textuelle et historique, voir VIRGILIO (1982).







85. Pour ce texte, voir aussi IvP II 251 ; LSAM 13. La datation du document à la période de peu postérieure à la fin de la dynastie attalide s'impose en raison de l'absence de toute référence au rôle régulateur du roi (cf. commentaire dans CGRN 206). L'hypothèse que la famille royale ne fût pas impliquée dans l'organisation du culte d'Asklépios, ce qui justifierait l'absence d'une référence au roi selon ceux qui soutiennent une date avant 133, est à rejeter à la lumière de IvP III 3, la base d'une statue honorifique érigée pour le prince Athénaios, fils d'Eumène II et agonothète de la deuxième édition des Sôteria et des Hérakleia (le premier nom renvoyant à l'épiclèse d'Asklépios Sôter). Il est donc probable que ce décret fut promulgué par les institutions civiques pour réglementer une prêtrise centrale dans la vie de la cité, lors de la phase politiquement et socialement instable qui suivit la guerre d'Andronique.





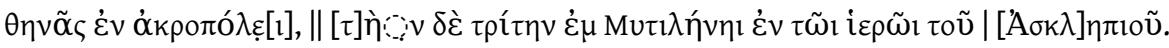

87. Aux indications fournies par Fränkel dans IvP I et II, on peut ajouter la lettre royale IvP I 163 (LE GUEN [2001], p. 243-250, nº 47) ; le texte stipule l'affichage d'une copie du document sur le mur du sanctuaire de Dionysos (III C, 9-10), erronément interprété par Fränkel comme celui de Pergame (ainsi aussi COARELLi [2016], p. 36-37), tandis qu'il est sans doute à identifier avec le sanctuaire de Dionysos de Téos, le plus important de la cité (cf. déjà OHLEMUTz [1940], p. 121). À Pergame, le texte fut affiché dans le sanctuaire d'Athéna, comme le suggère un passage très lacunaire du texte (IV C, 9-10).

88. Voir ROBERT (1937), p. 66-68, et (1984), p. 473-475.

89. Voir la liste offerte par BIELFELDT (2010), Appendice. Il en va de même pour les frises du grand autel, pour lequel cf. QUEYREL (2005), p. 27-28. Sur les murs de l'époque byzantine, voir KLINKOTT (2001).

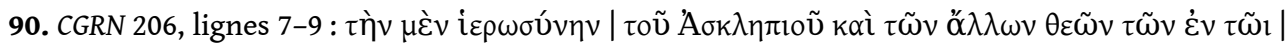



91. Une discussion de l'autre candidat pour le deuxième Asklépieion de la période hellénistique, le temple $\mathrm{R}$, amènerait aux mêmes considérations.

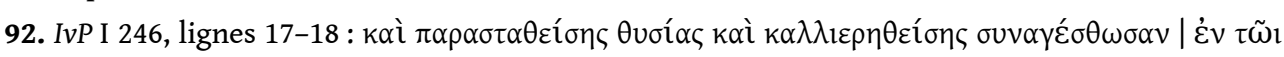



93. Des arguments similaires peuvent être proposés contre l'identification du temple $\mathrm{R} d u$ gymnase comme un Asklépieion intra muros. À ce propos, on peut observer que le bâtiment $\mathrm{H}$, 
situé directement au nord-ouest du temple $\mathrm{R}$, présente un podium en forme de $\Pi$ qui a été longtemps interprété comme la preuve de l'utilisation de cet espace comme une salle de banquet (DÖRPFELD [1910], p. 353), comparable à celle du bâtiment à la Podiensaal, le siège des Boukoloi, plus en haut sur la colline. Pourtant, la stratigraphie du site ne permet pas de dater le podium de l'édifice $\mathrm{H}$ avant l'époque impériale; de plus, l'interprétation de sa fonction fait toujours débat : cf. SCHWARZER (2004), p. 177 et (2008), p.121, proposant une utilisation comme socle pour supporter des statues plutôt que comme podium à banquet.

94. IvP I 246, lignes 19-20.

95. ROBERT (1984), développé par COARELLI (2016), p. 181.

96. IvP III 45-55. En revanche, l'argument avancé par MÜLLER (1992), p. 212, selon lequel la mention d'un seul prêtre d'Asklépios dans ces documents permettrait d'exclure l'existence d'un deuxième sanctuaire du dieu, n'est pas probant, car rien n'empêche qu'un prêtre desserve deux temples du même dieu.

97. Pour une analyse détaillée des éléments du texte imposant cette interprétation, voir CANEVA (2018).

98. COARELli (2016), p. 182, à la suite de virgilio $\left(2003^{2}\right)$, p. 107, 150. Cette interprétation est démentie par le fait que cette section comprend aussi (lignes 43-47) l'annonce, par le héraut sacré de la cité, des honneurs accordés par Pergame au roi à l'occasion ponctuelle de son retour de la guerre : une couronne à la valeur, un agalma dans le temple d'Asklépios et une eikôn dans l'agora. Il serait absurde de supposer que la cité stipule de concéder toujours ces mêmes honneurs à toute occasion où elle accueillera le roi.

99. COARELLI (2016), p. 179-184. Cette reconstruction est acceptée in toto dans le compte rendu de GRECO (2015), p. 327, qui note aussi la « giustificata ironia » de l'auteur à propos de la théorie des deux étapes de la rentrée d'Attale, comme elle fut développée par Robert (1984). Je partage l'opinion que l'interprétation de Robert est erronée, mais pour des raisons différentes de celles proposées par Coarelli.

100. Je signale à ce propos que l'identification de l'édifice à péristyle Haus $\mathrm{I}$, près de l'agora inférieure (DöRPFELD [1904], p. 116-120), avec le prytanée de la Pergame royale (COARELLI [2016], p. 186-191) est à rejeter à la lumière des fouilles récentes qui ont redaté le développement de la ville basse, y compris la deuxième agora, du $\mathrm{I}^{\mathrm{er}}$ s. av. J.-C. : cf. PIRSON (2017), p. 82-83, 92. De plus, il semble que, pendant les premières phases de son développement, l'agora inférieure fut caractérisée par une fonction exclusivement, ou au moins principalement, commerciale: cf. BIELFELDT (2010), p. 168-169 ; EMME (2013), p. 165-171; SIELHORST (2015), p. 144, 147 ; voir aussi DICKENSON (2017), p. 67-69, pour la tendance de certaines cités hellénistiques à développer une deuxième agora à la fonction spécialisée. On peut aussi exclure que le prytanée de la phase attalide se trouve dans l'un des édifices du versant de la colline - le bâtiment $\mathrm{H}, \mathrm{Z}$ ou le soi-disant Hestiaion; cf. SCHWARZER (2004) - qui auraient été hors des murs de la cité au moment où la charge du prytane fut intégrée dans les magistratures de Pergame, au IV siècle av. J.-C. Une localisation dans la cité haute, dans la partie est de l'agora (qui demeure toujours mal connue : cf. RHEIDT [1992] ; BIELFELDT [2010], p. 169-183; SIELHORST [2015], p. 141) ou dans ses alentours (cf. l'hypothèse de RHEIDT [2015], p. 303-304, proposant le soi-disant Temenos für den Herrscherkult) semble plus probable.

101. Cette indication est prise au sérieux par BURRELL (2004), p. 33, qui contra MÜLLER (1992), p. 214215, commente: "Though Müller would like to eliminate the current attribution of several temples in Pergamon to Asklepios, there may have been at least one such temple outside the Asklepieion, at least by Antonine times. The young Mark Aurelius made a metaphoric trip to the arx of Pergamon to entreat Asklepios for his teacher Fronto's good health ; though the journey may have been imaginary, that need not make a temple on the heights imaginary too ". Dans la même direction, cf. COARELLI (2016), p. 30-31. RENBERG (2016), I p. 120, n. 12 se limite à signaler le caractère métaphorique du voyage de Marc 
Aurèle, qui ne se rendit pas personnellement à Pergame, mais n'entre pas dans la question de la topographie du culte.

102. On peut rappeler à cet égardle règlement de pureté IvP II 264 (LSAM 14), datant du milieu du II $^{\mathrm{e}}$ siècle ap. J.-C. (cf. ci-dessus, n. 37). Trouvée en amont du gymnase, cette stèle concernait sans doute les cérémonies de pureté nécessaires pour accéder aux rituels ayant lieu dans le sanctuaire de la vallée: elle témoigne dès lors d'une multiplication des espaces visant à interpeller les nombreux pèlerins qui venaient visiter Pergame pour se faire soigner par le dieu, sans pour autant remplacer l'Asklépieion extra muros.

103. Cf. VENTROUX (2017), p. 76-82, à propos du succès du II $^{\mathrm{e}}$ siècle, suivi par un déclin assez rapide à partir du siècle suivant.

104. BURRELL (2004), p. 33. Sur ce temple, voir aussi RIETHMÜLLER (2005), I, p. 335-336 et (2011), p. 233 ; STROCKA (2012), p. 246-259, 271; MELFI (2016), p. 110-111. Sur l' évergétisme deRufinus, cf. veNTROUX (2017), p. 155-157, 210-213, 226, 271, 294-295.

105. La création d'un lieu de culte mineur d'Asklépios à l'intérieur d'une ville qui dispose déjà d'un grand Asklépieion hors-les-murs ne constitue pas un cas isolé : cf. RIETHMÜLLER (2005), I, p. 362-363. Cette savante observe que ce phénomène trouve aussi des parallèles dans d'autres cultes qui, à cause de leurs pratiques rituelles spécifiques, nécessitaient des structures particulières, pour lesquelles des sites extra-urbains se révélaient mieux adaptés; lorsque ce culte gagnait du succès, la cité qui l'hébergeait pouvait décider de lui donner aussi une place à l'intérieur des murs, pour marquer plus clairement le lien entre le sanctuaire extra-urbain et la cité elle-même.

106. Cf. ci-dessus, n. 28. En général, on renvoie à PALAmidis (2017) pour une étude récente de la documentation relative à la re-fonctionnalisation de lieux de culte après une phase d'abandon.

\section{RÉSUMÉS}

Cet article réexamine la documentation sur le sanctuaire extra-urbain d'Asklépios et le temple ionique de la terrasse du théâtre à Pergame. Ces deux cas d'étude permettent d'étudier les développements diachroniques du paysage sacré de la cité et de ses alentours pendant la « longue période hellénistique" (fin $\mathrm{du} \mathrm{IV}^{\mathrm{e}} \mathrm{s}$. av. J.-C. - début du $\mathrm{III}^{\mathrm{e}}$ ap. J.-C.). Cette analyse interdisciplinaire combine les sources textuelles, archéologiques et numismatiques pour étudier la distribution topographique des cultes pour les dieux et les chefs politiques, leurs relations et changements à travers le temps. Pour ce faire, plusieurs questions méthodologiques sont examinées : les critères permettant de distinguer la présence du temple d'un dieu des traces diffuses de son culte à travers le paysage urbain; la topographie de la dissémination et du remploi des textes et des artéfacts anciens et le type d'information qu'elle livre sur leur situation et fonction originaires; l'équilibre entre continuité et innovation dans les modèles d'intégration des cultes pour les chefs politiques dans l'espace et le temps sacrés de la communauté. L'article apporte un nouveau soutien à la thèse selon laquelle Pergame n'abrita jamais un deuxième Asklépieion à l'intérieur de ses murs.

This paper provides a reassessment of the evidence concerning the extra-urban sanctuary of Asklepios and the Ionic temple on the theatre terrace in Pergamon. These case studies allow for an investigation of the diachronic developments of sacred landscape in the city of Pergamon and its surroundings during the "long Hellenistic Age" (late 4th BC - early 3rd AD). The 
interdisciplinary analysis systematically combines textual, archaeological, and numismatic evidence in order to explore the topographic distribution of cults for gods and for ritually honoured rulers, their relationships, and their changes through time. In doing so, various methodological issues are dealt with: the criteria by which one can distinguish the presence of the temple of a god from the scattered traces of his worship across the archaeologically reconstructed city-scape; the topographic dissemination and reuse of ancient texts and artefacts and the type of information it can deliver with regard to their original location and function; the balance between continuity and innovation in the integration of cults for rulers within the sacred space and time of the community. Finally, this fine-grained analysis provides new support to the thesis that Pergamon never hosted a second Asklepieion within its city walls.

\section{AUTEUR}

\section{STEFANO CANEVA}

F.R.S.-FNRS - Université de Liège

ste.caneva@gmail.com 